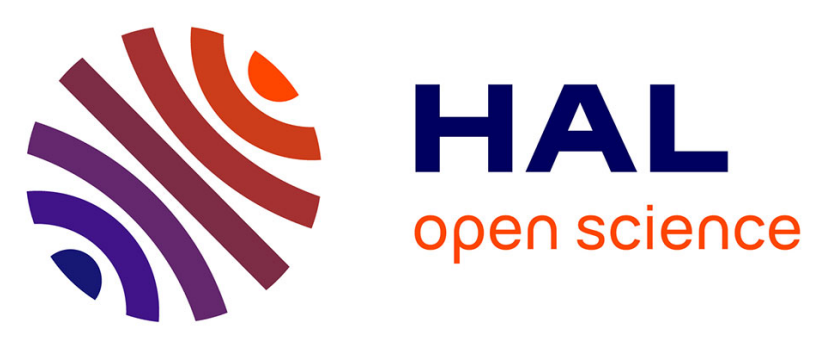

\title{
Calibration: A Conceptual Framework Applied to Scientific Practices Which Investigate Natural Phenomena by Means of Standardized Instruments
}

Léna Soler, Frédéric Wieber, Catherine Allamel-Raffin, Jean-Luc Gangloff, Catherine Dufour, Emiliano Trizio

\section{To cite this version:}

Léna Soler, Frédéric Wieber, Catherine Allamel-Raffin, Jean-Luc Gangloff, Catherine Dufour, et al.. Calibration: A Conceptual Framework Applied to Scientific Practices Which Investigate Natural Phenomena by Means of Standardized Instruments. Journal for General Philosophy of Science, 2013, 44 (2), pp.263-317. 10.1007/s10838-013-9231-7 . hal-01223197

\section{HAL Id: hal-01223197 https://hal.science/hal-01223197}

Submitted on 9 Feb 2022

HAL is a multi-disciplinary open access archive for the deposit and dissemination of scientific research documents, whether they are published or not. The documents may come from teaching and research institutions in France or abroad, or from public or private research centers.
L'archive ouverte pluridisciplinaire HAL, est destinée au dépôt et à la diffusion de documents scientifiques de niveau recherche, publiés ou non, émanant des établissements d'enseignement et de recherche français ou étrangers, des laboratoires publics ou privés. 
Authors:

Léna Soler, Frédéric Wieber, Catherine Allamel-Raffin, Jean-Luc Gangloff, Catherine Dufour, Emiliano Trizio

\section{Title:}

Calibration: a conceptual framework applied to scientific practices which investigate natural phenomena by means of standardized instruments

Running head (condensed title):

Calibration: a framework

Affiliations and addresses of the authors:

Léna Soler: Université de Lorraine, CNRS, Laboratoire d'Histoire des Sciences et de Philosophie - Archives Henri Poincaré, UMR 7117, 91 avenue de la Libération, Nancy, F54000, France.

Frédéric Wieber: Université de Lorraine, CNRS, Laboratoire d'Histoire des Sciences et de Philosophie - Archives Henri Poincaré, UMR 7117, 91 avenue de la Libération, Nancy, F54000, France.

Catherine Allamel-Raffin: Université de Strasbourg, IRIST (Institut de Recherches Interdisciplinaires sur les Sciences et la Technologie), 7 rue de l'Université 67000 Strasbourg.

Jean-Luc Gangloff: Université de Strasbourg, IRIST (Institut de Recherches Interdisciplinaires sur les Sciences et la Technologie), 7 rue de l'Université 67000 Strasbourg.

Catherine Dufour: Université de Lorraine, Institut Jean Lamour (IJL), UMR 7198 CNRS, Parc de saurupt, CS 50840, 54011 NANCY cedex.

Emiliano Trizio : Seattle University, 901 12th Avenue, Box 222000, 98122-1090, Seattle, USA

E-mail address, telephone and fax numbers of the corresponding author:

Corresponding author: Léna Soler

E-mail address: 1_soler@club-internet.fr 


\section{Abstract:}

This paper deals with calibration in scientific practices which investigate relatively wellunderstood natural phenomena by means of already standardized instrumental devices. Calibration is a crucial topic, since it conditions the reliability of instrumental procedures in science. Yet although important, calibration is a relatively neglected topic. We think more attention should be devoted to calibration. The paper attempts to take a step in this direction. The aims are two-fold: (i) to characterize calibration in a relatively simple kind of scientific practices; (ii) to provide conceptual and taxonomic tools of broader scope that help to get a better understanding of calibration in more complex cases and other kinds of scientific practices. In this purpose, indications are first provided about why a conceptual framework is needed for better understanding calibration. Second, a bibliographic survey of works dealing with calibration is attempted. Third, different tools, elaborated for a better understanding of calibration, are introduced. Fourth, we turn to the elaboration of what we call a "simple exemplar" of calibration, illustrated through the case of the calibration of an equal-arm balance. Fifth, the tools previously introduced, and the framework of the simple exemplar, are put to work on a more complex case of calibration (calibration procedures in X-ray experiments). This leads to show the work accomplished by the simple exemplar, and to emphasize features of more complex cases of calibration. Finally, we revisit and specify the nature, the status, the scope and the value of the proposed framework.

\section{Keywords:}

Calibration, standard, measurement, scientific instruments, measuring device, scientific practices 


\section{Introduction}

This paper ${ }^{1}$ deals with calibration, and is more especially focused on calibration in a certain kind of scientific practices, namely practices which investigate relatively well-understood natural phenomena by means of already standardized instrumental devices - for short, say UNSI practices (U as Understood, N as Natural, SI as Standardized Instruments).

\subsection{A crucial but neglected topic}

Calibration is a crucial but neglected topic. It is a crucial topic from an epistemological point of view, since it essentially conditions the reliability of instrumental procedures which play a major role in science. The outputs of measurement apparatuses are no more than marks deprived of a determined physical significance, unless the apparatuses in question have been correctly calibrated. New devices or techniques must successfully pass calibration tests (or tests akin to calibrations) in order to be recognized as sound candidates for further development, including broad diffusion in the scientific community and in diverse non-scientific social groups.

Yet although important, calibration is a relatively neglected topic (see section 2). This is probably because in many configurations at least, calibration is treated as a preliminary unproblematic procedure that precedes the 'main show' - namely, the characterization of objects of interest by means of what has been previously calibrated in this purpose. Under closer examination, however, calibration is rarely a straightforward matter. The study of calibration practices shows that even in the at-first-sight less problematic configuration, that is, in ordinary, relatively routine scientific practices, calibration procedures are often delicate, complex, and not obvious activities. This a fortiori holds for calibrations involved in more creative, not to say - borrowing a famous Kuhnian vocabulary - "extra-ordinary" or "revolutionary" scientific practices.

\subsection{Aims and structure of the paper}

Given that, we think that more attention should be devoted to the topic of calibration. The present paper attempts to take a step in this direction.

The aims are two-fold: (i) to characterize calibration in a particular, relatively simple kind of scientific practices, namely UNSI practices; (ii) to provide, beyond this particular simple case, conceptual and taxonomic tools of broader scope that also help to get a better understanding of what can be calibration in more complex cases and in other types of scientific practices (different from UNSI practices).

The article is structured as follows. First, we give indications about why we think a framework is needed (section 1.3) and how the framework has been constructed (section 1.4). Second, a survey of the works interested in science and knowledge that have dealt with calibration is attempted, and our own work is situated in the pictured landscape (part 2). Third, different tools, elaborated for the purpose of a better understanding of calibration, are introduced: (i) the four-question frame (section 3.1.1), which invites to specify the target, the presuppositions, the aim and the procedure of calibration; (ii) a rough mapping of scientific practices (section 3.1.3), which enables to situate the primary object of this article, calibration in UNSI practices, with respect to calibration in other kinds of practices, and on this basis, to motivate our choice to start with calibration in UNSI practices (3.1.4); (iii) a frame for the conceptualization of measuring devices (3.3), needed to get a fine-grain grasp of the classic target of calibration - which is, classically, a measuring device. The strategy we have followed is also explicated (3.2), in particular the "strategy of the simple exemplar" (3.2.2), which consists in the construction of an exemplar in the Kuhnian sense, intended to work as a benchmark and a compass for the analysis of instances of calibration. Fourth, we turn to the elaboration of the simple exemplar of calibration in UNSI practices, illustrated through the relatively simple and familiar case of the calibration of an equal-arm balance (part 4). Fifth, a more complex case of calibration in UNSI practices is examined (part 5): some calibration procedures in $\mathrm{X}$-ray experiments. The tools previously introduced, and the framework of the simple exemplar, are put to work for the analysis of this case. This leads to show the kind of work accomplished by the simple exemplar, and to emphasize some features of more complex cases of calibration that are not captured by the simple exemplar. Finally, as a conclusion, we revisit and specify the nature, the status, the scope and the value of the proposed framework.

\footnotetext{
${ }^{1}$ This paper is the product of a collective research project called "PratiScienS", launched by Léna Soler in 2007 in Nancy (France). PratiScienS stands for "Rethinking science from the standpoint of scientific practices". The aim of the project is to evaluate what we have learned about science from the practice turn in the science studies. One axis of the PratiScienS program is to characterize the procedures, reasoning, schemes of action, through which practitioners of the experimental sciences establish robust achievements, and calibration is part of these procedures. For further information about PratiScienS, see <http://poincare.univ-nancy2.fr/Activites/? contentId=6163\&languageId $=1>$.
} 


\subsection{A conceptual framework to find a way into the jungle of calibration activities and definitions}

In order to motivate the project of building a conceptual framework for calibration ${ }^{2}$, it is required to say a word about the process that led our research team to conclude that such a framework was needed, and more generally about the broader research program of which the present work is part.

It is through Allan Franklin's work that we first met calibration as an object of interest in philosophy of science. Franklin provides a repertory of what he calls "experimental strategies", and calibration is one of these strategies. At the beginning, our aim was to consider Franklin's repertory of experimental strategies in a critical perspective, and to give a refined characterization of recurrent schemes of action through which scientists institute robust results (i. e., what they take to be reliable results). To advance progressively with respect to this aim, we decided to start with calibration. Was not calibration, on the one hand a preliminary inevitable step, required in any experimental activity aiming at the establishment of robust results, and on the other hand a not too complicated activity, at least compared with subsequent scientific investigations by means of previously calibrated devices? We bet this was indeed the case, so we took calibration as the starting point of our inquiry on experimental schemes of action. But we rapidly discovered that what was at stake was not so simple (to say the least), and at the same time, much more interesting than we have anticipated. As a result, calibration became an object of research per se.

For our inquiry on calibration, we relied on two kinds of materials: on past and present case studies available in the literature; but also, and actually first of all, on a more direct observation of today science as it is performed in practice. The analysis of 'today science as it is performed in practice' was possible, since two members of our team were in a position to provide such resources. A first set of examples of actual practices was provided by Cathy Dufour, who had been trained as a physicist and worked, for more than twenty years, in an experimental laboratory of condensed matter physics in Nancy, the Institut Jean Lamour (hereafter IJL) ${ }^{3}$. A second set of examples came from ethnographic studies conducted by Catherine Allamel-Raffin in the field of astrophysics and pharmacology 4 .

Relying on these materials, and interacting with physicists, astrophysicists and pharmacologists, we rapidly came to realize that the kinds of activities called "calibration" were not a set of obviously homogeneous activities. This heterogeneity was strikingly reflected in the instances of current scientific practices on which we relied as the material of the present inquiry. Different procedures were called "calibration" by the pharmacologists, astrophysicists and solid state physicists we studied. Moreover, some activities we were intuitively inclined to identify with calibration were not viewed as calibration by some practitioners.

To give just one example, the pharmacologists we studied did not consider the creation of a standard curve (in order to measure quantities of cytokine) as a procedure of calibration, but, rather, as the first part of their experiment ${ }^{5}$. Whereas according to the intuitions of the members of our research team, such a procedure was, without discussion, a case of calibration - since to perform a standard curve amounts to establish a reference scale (here with respect to quantities of cytokine), thanks to which the outputs of subsequent measurements can be converted into determined values of quantities of cytokine. Astrophysicists with whom we interacted operated with the same intuitions as ours. The procedure which, in astrophysics, can be seen as the equivalent of the etalon scale in pharmacology, starts with the choice of a set of celestial objects whose physical properties are

\footnotetext{
${ }^{2}$ We are grateful to Sally Riordan (one of the reviewers of this paper, which agreed to be identified after the reviewing process), to have insisted on the need to motivate the project more explicitly, so as to highlight its value better.

${ }^{3}$ Very sadly Cathy Dufour, who is one of the co-authors of the present paper, who has been a crucial contributor of our research group, and who was also a very dear friend, prematurely passed away in March 2011 at the age of 46 years. This reference to her contribution is an occasion to pay homage to her.

${ }^{4}$ Pharmacological observations were conducted at the laboratory «Inflammation et environnement dans l'asthme» (EA 3771), Faculté de Pharmacie de Strasbourg, France. Astrophysical studies, at Center for Astrophysics (CfA), Harvard, Cambridge, USA.

${ }^{5}$ At the laboratory under scrutiny, scientists produce the standard curve by means of the ELISA test (Enzyme linked ImmunoSorbent Assay). This left them with a correspondence between determined quantities of cytokine and instrumental outputs. They explicitly deny that this procedure is a calibration, and say: "When we produce a standard curve, it's in fact the first step of an experiment, it's included in the experiment, it's a part of an experiment” (Interview of FP, 03/03/2008, « Inflammation et environnement dans l'asthme » (EA 3771), Faculté de Pharmacie de Strasbourg, France, our translation from the French).
} 
already well-known. The objects in question are used to determine the relation between their known properties and the outputs of astrophysical instruments, so that when the same instruments are applied to unknown celestial objects, their outputs can be converted into reliable information about the phenomena under interest. Astrophysicists call "calibration sources" the well-known celestial objects which serve as points of reference in this procedure, and they identify the whole procedure with a calibration ${ }^{6}$.

We also came to realize that in the science studies literature ${ }^{7}$, the few works which made a central place to calibration rarely attempted to define calibration, and did not always rely on similar conceptions of calibration (see part 2). All in all, we rapidly felt lost at the heart of a dense and rich jungle - and we bet that anyone aiming at an understanding of what calibration is and what work calibration accomplishes in scientific practices would be subject to the same experience. In such a situation - which is perhaps typical of what holds for a relatively unexplored field - some order has to be introduced. This is precisely the function of the conceptual framework we are going to present in this article. Our ambition is to propose a framework able to play the role of a useful compass for researchers interested in the topic of calibration. We are confident that the framework will help these researchers to find one viable way - certainly not the only tractable path but at least one among other possible ones - into the 'jungle of calibration'. At the end of this article (section 6), once the main pieces of the framework will have been presented, and once the framework will have been put to work in relation to concrete practices of calibration performed at the IJL, we will articulate more precisely what we mean by a conceptual framework, what insights it has brought so far, and what insights it could bring applied to other cases not explored here.

\subsection{Genesis of the framework}

A word about the genetic process through which our conceptual framework has been generated in concreto will help to get a first sense of its nature and its potential value.

We started with a set of examples of activities candidate to calibration, i.e., activities that could possibly be identified with calibration for a reason or another - because the scientists with whom we interacted considered them as calibration; or because scholars in the science studies treated them as calibration; or because our own intuitions, as analysts of science, led us to assimilate them to calibration. As mentioned section 1.3., most of these examples were provided, on the one hand by the activities performed by C. Dufour and her colleagues at the IJL, and on the other hand by the astrophysical and pharmacological practices observed by C. Allamel-Raffin as an ethnographer of science. In order to find a path into the jungle of calibration, we attempted, starting from this diversified set of examples of practices candidate to calibration, to grasp their very nature, to identify significant differences between them, and to examine the possibility of some common core features between them. In other words, we attempted to construct a conceptual framework which would help to compare, classify and provide a better understanding of the activities under scrutiny.

Numerous successive versions of conceptual frameworks have been built in the course of our inquiry. The prism of each version shed a new light on our examples, but at the same time, new kinds of difficulties appeared, with which we had to cope through modified conceptualizations. So, the framework we are going to present is the result of a long and sinuous maturation process, constituted of a back and forth movement between our examples of calibration and multiple attempts to understand and classify them. Yet, the work invested in the clarification of the encountered difficulties and in the construction of more and more satisfying solutions is difficult to perceive in the end-product. Contemplating the final result apart from the process of its constitution, this final result might appear straightforward: the characterization of calibration provided in part 4 might appear obvious, and thus perhaps useless or uninteresting. We will come back to this point at the end of this paper, section 6.3.

\section{Calibration in the science studies: a survey}

Since the topic of calibration is in a so preliminary state, we think it might be useful to offer a view of how calibration has been dealt with so far in the science studies broadly understood (see note 7). This will enable us to introduce, without any pretention to be exhaustive, the main references which, according to us, provide interesting elements regarding the topic of calibration - even if calibration is not the primary object of interest of most of these works.

\footnotetext{
${ }^{6}$ Is this disparity of intuitions about calibration between pharmacologists and astrophysicists the manifestation of the fact that calibration is 'science dependent'? We leave the question open.

${ }^{7}$ All along this paper, we use this expression "science studies” in a very permissive sense, intended to encompass all studies that take science in general, or one particular science, as the object of their inquiry, whatever approach, method and orientation is preferred. This includes philosophy of science, history of science, social studies of science, conceptual analysis such as the ones of the International Vocabulary of Metrology (see part 2), and any imaginable hybrid of these.
} 
We map this new terrain by addressing three questions: the target of calibration; the definition of calibration; and the perspective in which calibration is called for. The answers attempt to take into account and relate many different domains and strands: history and philosophy of science, philosophy of experiment, social studies of science, philosophy of biology, philosophy of economics, metrology... as well as descriptive versus normative approaches.

\subsection{The target of calibration}

Let us start with the issue of the target of calibration - which is, as we will see section 3.1.1, one element of the frame through which we propose to analyze calibration. The question is: when we scrutinize both the science studies literature and scientists' ways of talking, what kinds of item appear to be the object of calibration activities?

\subsubsection{The traditional target: material instrumental devices}

Most of the time, and classically, the target of calibration is a material instrumental device, and often a measuring instrument.

Let us give a selection of examples of this type in the historical, philosophical and sociological studies of science. The target of calibration corresponds: to a spectrometer in Franklin (1997) (as well as to many other complex experimental devices); to thermometers in Carnap (1995 [1966]), Sibum (1995), and Chang (2004); to electron microscopes in Rasmussen (1993); to gravity-waves detectors in Collins (1985; 2004); to a chromatographer in Livengood (2009); to machines dedicated to the measurement of body composition in O’Connel (1993); to CO-CO2 analyzers in Mallard (1998); to a measuring instrument in general in Buccianti et al. (2009)...

In relation to the claim that the target of calibration is commonly identified with a material instrumental device, let us report a testimony issued from the studies of 'science in the making' that have fed our research on calibration. After having discovered disparities in the way the scientists with whom we interacted used the term "calibration", we tried to clarify the underlying conceptions of calibration involved. As a result, it appeared that for contemporary pharmacologists (or at least the one we met), the fact that the target is a material instrument worked as a necessary condition to identify a given type of measurement with a calibration. As one pharmacologist tells us: "We talk about calibration only when we calibrate an instrument such as a pipette, a pH

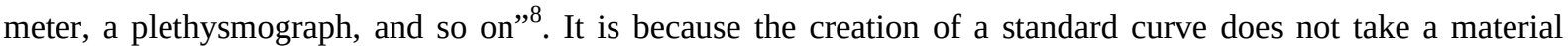
instrument as its target, that pharmacologists of this lab do not identify this procedure with a calibration procedure, as reported in section 1.3 .

\subsubsection{Stretching the target of calibration}

Calibration is, widely and classically, thought of as one way of controlling material instruments. However, besides the latter widespread traditional use of the calibration vocabulary, other, extended uses appear to hold. Let us attempt a brief survey of other kinds of target found in the literature. The target of calibration can be extended to:

Immaterial conceptual devices such as mathematical procedures: see Boumans (2004), who assimilates mathematical procedures used in the social sciences to measuring "non-material instruments"; or see Franklin (1997), who is ready to extend the target of calibration to the procedures of analysis - including computer analysis, cuts in the data, etc. - which mediate the conversion of raw data issued from the experimental device into claims about natural phenomena.

Models, for instance economic models (Boumans 2007), or biochemical models (see Ramsey (2007), who deals with models in protein chemistry).

Simulations (Boumans 2006; Werker and Brenner 2004).

Material but not artifactual objects, such as living beings: populations of organisms in the laboratory (see Skipper (2004), who discusses the calibration of populations of organisms in artificial selection experiments); or human beings, for example scientists considered as instrument-readers, taking into account the possibility that different practitioners might read in different possible ways (Woodward 1989), or for instance ordinary people considered as measuring systems in relation to quantities related to human perception (see for example Rossi et al. 2005), who discuss calibration in the context of panel or jury testing), or to take another last example, scientists considered as more or less trustful sources of scientific information that have to be calibrated (Andersen 2013).

\footnotetext{
${ }^{8}$ Interview of FP by CAR, 03/03/2008, « Inflammation et environnement dans l'asthme » (EA 3771), Faculté de Pharmacie de Strasbourg, France (our translation from the French).
} 
Authors who apply calibration to extended targets of the latter kinds are often well-aware that their use involves a 'stretching' with respect to the classical target identified with a material instrumental device; their decision to stretch the target is often deliberate and explicit. For instance, Franklin talks about an "extended sense" of calibration or of calibration "more broadly construed" (Franklin 1997, 33) when he turns from the calibration of material apparatuses to the 'calibration' of data analysis. Still more explicitly, Ramsey (2007, 310) writes: "Usually, we think of calibration as something we do to instruments. [...] However, calibration can involve any scientific apparatus, not just scientific instruments". Under the general notion of "scientific apparatus”, Ramsey includes, in addition to material instrumental devices (i.e., "scientific instruments”), other kinds of 'devices', such as equations (for example in quantum chemistry), models, and populations of organisms (Ramsey makes here a reference to Skipper (2004)).

\subsection{The definition of calibration}

Among the works which devote substantial developments to calibration - including those in which calibration is not the primary subject of the inquiry - very few of them provide an explicit definition of calibration. In what follows, we start from two available explicit definitions, and extract their common core. Next, we reconstruct, from works that do not define calibration but use the term, another definition which involves aspects not encompassed by the previous explicit definitions.

\subsubsection{Franklin's definition of calibration}

The most widely used definition of calibration is the explicit definition given by epistemologist Allan Franklin (Franklin 1986; 1990; 1997).

As already mentioned in section 1.3., Franklin gives to calibration the status of an "experimental strategy", used by scientists to legitimate the reliability of experimental achievements. In such a perspective, Franklin defines calibration as "the use of a surrogate signal to standardize an instrument" (Franklin 1997, 31). The signal is a "surrogate", in the sense that it is used as a substitute for the unknown phenomena that experimenters aim to investigate. The "surrogate signal" is a signal of already-known properties. The logic of the strategy is the following: "If an apparatus reproduces known phenomena [i.e., the known characteristics of the surrogate signal], then we legitimately strengthen our belief that the apparatus is working properly and that the experimental results produced with that apparatus are reliable” (Franklin 1997, 31).

Franklin's definition in terms of the use of a surrogate signal works as a reference. It is explicitly mentioned and exploited in many works interested in calibration, including works in which the target of calibration is extended beyond material instrumental devices (see notably Boumans 2004; 2007; Harris 2003; Ramsey 2007; Rasmussen 1993; Skipper 2004; Woodward 1989).

\subsubsection{The VIM's definition of calibration}

A second explicit definition, not frequently mentioned in the literature of philosophy, history and social studies of science - with the notable exception of the papers of Boumans and Tal (2011) - is offered in the International Vocabulary of Metrology (VIM). This definition is interesting to consider, since the VIM is the result of complex negotiations between metrological experts issued from diverse fields and trained in relation to diverse backgrounds, and since the central aim of the VIM is to provide a uniform vocabulary of international scope about measurements of all kinds in any scientific discipline.

The last edition of the VIM (2008) defines calibration as an "operation that, under specified conditions, in a first step, establishes a relation between the quantity values with measurement uncertainties provided by measurement standards and corresponding indications with associated measurement uncertainties and, in a second step, uses this information to establish a relation for obtaining a measurement result from an indication" (VIM 2008, 28). An "indication" is the "quantity value provided by a measuring instrument or a measuring system” (VIM 2008, 37).

The 2008 definition of the VIM involves two steps (say step 1 and step 2). In the step 1, a "measurement standard" is used. A measurement standard is an object of known properties. Step 1 consists in performing different measurements with the same measuring instrument, using different measurement standards characterized by different known values, and to record the "indications" (measured-values) delivered by the instrument in each case. The result is often presented as a curve which plots the measured-values obtained as a function of the values associated with the measurement standards. Since the values associated with the measurement standards are predetermined and known in advance, the measured-values provided by the instrument can be compared to them. Taken into account the uncertainties, the first step therefore enables to estimate the difference between the taken-for-granted values of the measurement standards and the values actually obtained. Then, in step 2, this information can be used, in subsequent measurements performed on some 
unknown phenomena under investigation, in order to convert the indications delivered by the instrument into correct values of the quantities under interest.

\subsubsection{A first sense of calibration: calibration by means of an object of known-properties}

Let us now compare Franklin's and the VIM's definitions of calibration. The "measurement standard” mentioned in the VIM's definition is equivalent to Franklin's "surrogate signal”. True, the VIM probably primary targets measurement standards that have been produced and certified by professional metrologists (let us call them "metrological measurement standards"), whereas the idea of a "surrogate signal" can be understand in a broader sense, including the use of any phenomenon assumed to be sufficiently well-known to play the role of a takenfor-granted reference with respect to some purpose. As Boumans writes: “Franklin's (1997) discussion of calibration as one of the epistemological strategies for the establishment of the validity of experimental results is useful for our discussion of accuracy because this strategy does not presuppose a standard" (Boumans 2004, 230, our italics). In this quotation, "standard" is meant in the sense of what we have called "metrological measurement standard", that is, an object inserted in a metrological traceability chain ordered in terms of degrees of precision. To the extent that Franklin's formulation does not necessarily implies a metrological measurement standard, it is more general, and this might be one reason why authors like Boumans and others, who attempt to stretch the target of calibration beyond material instruments of the natural sciences, frequently rely on Franklin's definition.

Beyond this difference, however, Franklin's “surrogate signal” and the VIM's “measurement standard” play the same role. In both definitions, the point is to make measurements on an 'object' of already-known properties (in a broad sense of the term 'object', not necessarily identified with a material solid). Furthermore, the same logic underlies Franklin's definition and step 1 of the VIM's definition, namely: since the 'correct results' of the measurements performed by means of the instrumental device under calibration on the 'object' of already-known properties is pre-determined, it is thus possible to evaluate the extent to which the result actually obtained differs from the taken-to-be-correct result. On this basis, the reliability of the instrument can be assessed.

True, Franklin's definition is not formulated into two steps. But it could have been, as it is obvious from Franklin's examples. In particular Franklin writes that calibration can "provide a numerical scale for the measurements or for a numerical correction" (Franklin 1997, 33). To apply a numerical correction to the measured-values delivered by the apparatus under calibration in subsequent measurements, would amount to perform an operation of the type described in the step 2 of the VIM's definition.

We conclude that the two definitions under discussion present a common core. This common core is: to compare the known-in-advance value of a property of an 'object' with the measured-value obtained for this 'object' by means of the measuring instrument under calibration; next, to use the result of this comparison to assess the reliability of the instrument and to correct the indications subsequently obtained when the same measuring instrument is applied to new, unknown objects. Let us call this conception of calibration: calibration by means of an object of known-properties.

\subsubsection{A second sense of calibration: calibration in relation to measurement scales}

A second sense of calibration - related to the previous one but sufficiently different to be treated apart - can be reconstructed from several works which, at a point or another of their developments, are led to talk about calibration but do not provide any explicit definition of calibration. This sense of calibration has to do with measurement scales.

This second sense of calibration is for instance involved in Carnap's famous 1966 book, An Introduction to the Philosophy of Science, chapter 6 (Carnap 1995 [1966]). The aim of this chapter is to analyze quantitative (or numerical) concepts. For that purpose, Carnap examines how “empirical criteria are established” (Carnap 1995 [1966], 53), which give meaning to quantitative concepts and enable to assign numerical values to them. He takes measurements of temperature with thermometers as an illustration. Calibration is mentioned in this context, in relation to scales of temperature.

Having explained how the magnitude "temperature" can be numerically specified through the construction of a given scale of temperature - for example the centigrade scale -, Carnap turns to comparison between scales. Considering the Fahrenheit, absolute (Kelvin) and centigrade scales, he writes: "The centigrade and Fahrenheit scales may be thought of as variants of the absolute scale, differing only in calibration and easily translated to the absolute scale” (Carnap 1995 [1966], 67, italics added). The three scales differ in calibration in the sense that "other states of substances are chosen for the zero and 100 points" (Carnap 1995 [1966], 66). Thus here, calibration concerns the choices that define one particular scale of temperature (choices of two fixed points, etc.). Calibration refers to some (partly conventional) decisions related to the concrete procedure through which some determined numbers are attributed to a given magnitude (here the temperature). 
Leaving Carnap's particular text and taking into account other writings, we can say that in relation to scales, calibration - the act of calibration, or equivalently the verb "to calibrate" - name two types of actions.

(i) In some contexts, calibration names the genetic process of construction of a given scale and the choices finally retained (for the zero, etc.), by some scientists or groups of scientists, in a given episode of the history of science. In such case, "to calibrate" means "to elaborate a new scale and to take the decisions that go with such task”. We find this sense, for example, in some of Chang's and Sibum's historical works, which, as Carnap's, discuss thermometry. Chang shows how hard it can be to determine the two fixed points that are needed to define a scale of temperature, as well as the precise form of the scale (see Chang (2004), notably chapters one and two). Sibum directs the attention on the uniformity of the inner diameter of the thermometer tube, which can be a problem in practice, and he analyses how Joule ingeniously dealt with this problem (Sibum 1995). Chang and Sibum explicitly use the vocabulary of calibration in their discussion. Although they do not define calibration in this context, we can reconstruct their position as involving sense (i).

(ii) In some other contexts, calibration names the use of an available (already established) scale. In such case, "to calibrate" means "to use this taken-for-granted scale as a reference for certain purposes". The just-cited works of Chang and Sibum also involve this sense. For example, Chang mentions "the fact that Wedgwood did calibrate his thermometric scale with the Fahrenheit scale" (Chang 2004, 127). In the same vein, Sibum writes: "In order to compare his measurements with those of contemporary researchers he [Joule] very often brought foreign thermometers into his laboratory (...) to calibrate them against his own" (Sibum 1995, 101). Some instances of what Rasmussen names "calibration against different instruments or methods" (Rasmussen 1993) can also be interpreted as occurrences of sense (ii).

\subsection{Three (not mutually exclusive) perspectives on calibration}

Why are scholars of science studies interested in calibration? For what purposes, in what perspective, do they mention calibration? We distinguish three perspectives or trends: epistemological, socio-historical and analytic. The three perspectives are conceptually distinct, but from a logical point of view, they are not necessarily mutually exclusive, and from an empirical point of view, they are sometimes involved and articulated, in practice, in one and the same work.

\subsubsection{An epistemological perspective aiming at the adjudication of reliability issues}

A first perspective is epistemological and revolves around the justification of reliability. The central issue is "the importance of calibration in establishing the reliability of scientific knowledge" (Rasmussen 1993, 229). In our categories, the issue is the reliability of the target of calibration - material apparatuses and their outputs, and by extension, as we have seen, possibly many other things such as models, simulations, data analysis, and so on. The issue is to determine if, and to what extent, calibration is one way to warrant, justify or at least support, reliability claims about the target (and when the target is an instrument, reliability claims about the data obtained with this instrument).

This perspective seems to be the most represented one. It must be added that reliability is probably an always involved horizon, even when reliability issues are not explicitly placed on the front line. In this perspective, the emphasis is on the function of calibration. The primary question is: what, exactly, is the power of calibration with respect to the justification of the reliability of its target? Which in turn leads to investigate in more or less details questions such as: what is calibration? What are the criteria to conclude that a given target is indeed wellcalibrated? What kind of work do calibration procedures accomplish? In brief, the aim is to evaluate the nature and the force of the support calibration provides, in terms of reliability, to the target of calibration.

This epistemological perspective is the one within which Franklin has introduced calibration in the science studies. In this vein, calibration figures among the "epistemological strategies" (see for example Franklin 1997, 1, italics added) listed by Franklin in his "epistemology of experiment”. Relying on Franklin's proposal, several other scholars have analyzed calibration in the same perspective. Boumans (2004), Ramsey (2007), Rasmussen (1993), Skipper (2004), Tal (2011), or Woodward (1989) can be seen as examples. These authors have discussed calibration as a potential way to support the reliability of diverse kinds of material or immaterial scientific instruments, in relation to different areas of the natural and the social sciences.

The same epistemological perspective focused on justificatory issues about reliability, also constitutes the background of the famous Franklin-Collins debate about the so called "experimenters' regress" (Collins 1985; 2004). In relation to calibration, the debate revolves around the question of knowing whether or not the "experimental strategy of calibration" (in Franklin's terms) is able to stop the experimenter's regress. Franklin argues in favor of a positive answer, at least in typical scientific configurations, whereas Collins advocates a negative answer. Franklin and Collins disagree about the power of calibration procedures, as well as about the 
kinds of factors (characterized as “epistemological” versus "social”) involved in scientists' judgments about whether or not a given instrument is indeed correctly calibrated and hence reliable.

To the extent that the aim is to settle justificatory issues, this perspective can be characterized as normative. Accordingly, the above-mentioned works can be characterized as in part normative (only in part, because the epistemological conclusions of these works often rely on historical accounts of scientific episodes that are assumed to be descriptive in status and descriptively accurate).

\subsubsection{A socio-historical perspective aiming at the description of the standardization of instruments at a more or less broad scale}

By contrast to the normative, epistemological perspective primarily directed toward justificatory issues, we can identify a second perspective, animated by a more 'purely' descriptive spirit, within which calibration is studied as a socio-historical object. The aim, in this socio-historical perspective, is to provide an descriptively accurate account of the (often complex) routes through which instrumental devices, techniques and objects of all sorts have, historically, acquired the status of well-calibrated and hence reliable items, either at a local level (at the scale of one individual or small groups of scientists), or at a global, possibly international level.

A relatively important corpus of socio-historical studies dedicated to the local conception and realization of new instrument devices, which, at a point or another of their developments, are led to mention calibration, fall within this second perspective. In this vein, we can mention, for instance, Bourget, Licoppe and Sibum (2002), Chang (2004), Chang and Yi (2005), Heering (2005), Licoppe (1996), Livengood (2009), Schickore (2007), Sibum (1995). In many of the studies of this corpus, however, calibration is not the central topic: it is considered incidentally, or among other, equally or most important issues (for example, the theoretical principles on which the explanation of the instrument rests). We could perhaps also include, as examples of the socio-historical perspective, the 'historical part' of the epistemological studies mentioned section 2.3.1 - providing we are ready to assume that the descriptive-historical stratum can be separated, and considered apart, from the epistemological-normative contribution.

Other works conducted in the socio-historical perspective involve calibration in relation to standardization at a broader scale and professional metrology. Calibration is a central task in professional metrology. Calibration in metrology has to do with the conception, realization, standardization and spreading at a large scale (ideally an international scale) of instruments, measurement standards, and units. Mallard (1998) or O'Connell (1993) can be cited as works conducted in a socio-historical perspective, which mention calibration in relation to professional metrology and large-scale standardization - O’Connell talks about "the creation of universality" (in the title of the article), Mallard about "how results and discoveries that emerge in a culturally laden context can be made universal” (Mallard 1998, 573).

\subsubsection{An analytical perspective aiming at a systematic conceptualization of calibration}

Finally, a third perspective on calibration can be introduced: the analytical perspective. Considered at the most ambitious level, the primary aim of the analytical perspective is the achievement of a systematic, fine-grained and comprehensive conceptualization of calibration.

As far as we know, only two writings explicitly and deliberately examine calibration in an analytical perspective: the VIM (VIM 2008), and one article written by authors involved in the elaboration of the VIM (Buccianti et al. 2009), which intends to explain and specify some aspects of the definition of calibration provided by the VIM ${ }^{9}$. Admittedly, a few number of the works conducted in the epistemological and socio-historical perspectives provide some insights about what calibration is (or may be). However, none of these works go very far in this direction, and in any case, none of the corresponding characterizations can be considered as systematic, finegrained and sufficiently complete conceptualizations of calibration (which is not a reproach to these works, since to achieve such a characterization is not their aim). Carnap's perspective, in Carnap (1995 [1966], chapter 6), can be characterized as analytic, but as we have seen section 2.2.4, the subject matter of Carnap's chapter is not calibration, and in this chapter, calibration is not the target of any specific analysis. Since the VIM is the only available attempt to provide a systematic conceptualization of calibration, and since this is also our aim in the present article, let us specify the distinctive features of the analytic perspective of the VIM. This will help, in the next section, to situate and motivate further our own project.

First, the analytic perspective of the VIM is a normative perspective, intended to standardize the vocabulary and the concepts at an international scale, as it is clearly stated at the beginning of the document. "This Vocabulary is meant to be a common reference for scientists and engineers - including physicists, chemists, medical scientists - as well as for both teachers and practitioners involved in planning or performing measurements, irrespective of the level of measurement uncertainty and irrespective of the field of application. It is also meant to be a

\footnotetext{
${ }^{9}$ Mari and Sartori (2007) also contains some insights about calibration in an analytic perspective.
} 
reference for governmental and intergovernmental bodies, trade associations, accreditation bodies, regulators, and professional societies.” (VIM 2008, 1). Calibration is defined in order to standardize what calibration has to be in different socio-techno-scientific contexts. In this horizon, second, the aim of the VIM is to list and define the "basic and general concepts" of metrology, and metrology is understood as the "science of measurement and its application" (VIM 2008, vii). The concepts of metrology that have to be listed and defined are "basic and general", in the sense that they are intended to be trans-disciplinary valid. Such project appears realizable because the VIM takes "for granted that there is no fundamental difference in the basic principles of measurement in physics, chemistry, laboratory medicine, biology, or engineering” (VIM 2008, vii). Accordingly, the definition of calibration proposed by the VIM is very general - and could be specified in regard to more specific classes of scientific configurations. Moreover, third, the definition is not very developed, and could be developed further - which is an obvious consequence of the fact that calibration is only one of the multiple concepts that the VIM attempts to define. Furthermore, fourth, the VIM is only concerned by measurements which use already standardized instrumental devices, measurement standards and units. Consequently, the VIM’s definition could be completed to encompass calibration in more innovative practices.

\subsubsection{Situation of our work on calibration with respect to others}

We have distinguished three perspectives, associated with different purposes, according to which the topic of calibration is discussed in the science studies literature. Let us now situate our own work with respect to these three perspectives. On this basis, we shall be in a position to motivate it further (in addition to what has already been developed in section 1.3) and give a more precise idea of its object.

In the present article, our perspective is analytic - even if the ultimate aim of our research program about calibration is epistemological (see section 1.3). Thus, our approach differs from epistemological discussions centered on justification of reliability and from socio-historical studies intended to describe how instruments have been stabilized in particular historical episodes. Our approach intends to provide a systematic conceptualization of calibration. Our analytic aim, expressed at the more general level, is to achieve a systematic, detailed and reasonably complete characterization of calibration. The existing conceptualizations are, according to us, far from being sufficient. We have indicated why in the previous section, focusing on the only available systematic characterization of calibration, namely the characterization of the VIM.

In this paper, we take a step in the direction of achieving our general aim. To tackle the problems one by one, we restrict ourselves, as a starting point, to a relatively simple and not too problematic case, calibration in UNSI practices - practices in which scientists use already well-mastered devices for the purpose of the investigation of relatively well-known types of phenomena. This circumscribed object of study - calibration in UNSI practiced considered in an analytic perspective - is different from what is most of the time studied in the literature under the heading of "calibration". Calibration in UNSI practices is different from calibration in professional metrological practices (understood as standardization at the international scale), and different from local practices of establishing new measurement scales or new instruments. Calibration in UNSI practices, as it will appear, encompasses instances of Franklin's and the VIM's definition, as well as instances of calibration against a given established scale. But beyond this partial intersection, first, our analytic perspective differs from Franklin's epistemological one, and second, the characterization of calibration we offer in this paper is more elaborated than the definition of the VIM.

In the next part of this article, our perspective will be further clarified, through the presentation of some of the strategic options we have adopted and of the tools we have elaborated for the purpose of the investigation of calibration.

\section{Tools and strategy}

\subsection{A first set of tools}

\subsubsection{Four questions to investigate calibration}

In order to characterize calibration, it proves helpful to investigate four questions.

1) The target $\boldsymbol{T}$ of calibration: What kind of thing can be the object $O$ of a calibration?

2) The presuppositions Ps of calibration: what is taken for granted, which delimitates what is not granted and has to be checked and controlled?

3) The aim of calibration applied to the object $O$ under presuppositions Ps.

4) The procedure of calibration: the nature of its structural elements and the kind of logical stages through which the aim of calibration is achieved. 
The question of the target of calibration has already been introduced above, and applied as an analyzer to the literature related to calibration (see 2.1). As a result, it appeared that the target of calibration can be identified with heterogeneous kinds of objects, such as material apparatuses and immaterial mathematical procedures. This heterogeneity suggests the possibility that different targets might go with different types of calibration activities. In any case, we cannot presuppose without examination that calibration activities are not target-dependent. A complete analysis of calibration should examine, for different types of calibration targets, if it makes a difference, and if so, should specify the kind of difference it makes. In this paper, however, we restrict ourselves to the most common case: the case in which the target of calibration is a material instrumental device.

The four questions are part of the framework we propose in order to achieve a fine-grained grasp of calibration. We will see below how the four questions apply to the case of calibration in UNSI practices, and what the answers are in this case (see part 4). However, the intended scope and fecundity of the four-questions frame go much beyond the particular case of calibration in UNSI practices. We will come back to this point in the conclusion of this paper, section 6.3.

\subsubsection{What are UNSI practices?}

When attempting to introduce some order into the network of activities that can be identified with calibration, it proves useful to distinguish types of scientific practices with respect to which calibration takes different forms and is more or less problematic. In this article - to repeat - we concentrate on calibration in one particular type of scientific practices, abbreviated as UNSI practices. It is thus needed to specify in more details what UNSI practices are. Since the specification of what UNSI practices are requires the delimitation of what they are not, other types of scientific practices will inevitably have to be introduced into the picture.

UNSI practices investigate well-understood domains of natural phenomena with already standardized instrumental devices. More precisely, UNSI practices are characterized by three structural distinctive features.

(i) The primary target of the inquiry is the natural world. The aim is to acquire knowledge about nature (hence the "N" of UNSI, as Nature). This differentiates the practices in question from scientific practices directed toward the means of scientific inquiry - instrumental devices and techniques -, such as professional metrological practices or practices devoted to the local invention of a new type of instrument.

(ii) The natural phenomena under inquiry are explored by means of already standardized instrumental devices (hence the "SI" of UNSI, as Standardized Instruments). In other words, the means of the inquiry are already well-designed and widely entrenched in some scientific communities. They are already theoretically wellunderstood and practically well-mastered. Practitioners of UNSI practices are users of socially well-mastered instrumental devices. This distinguishes UNSI practices from practices in which actors are designers of new instrumental devices.

(iii) The kinds of natural phenomena under inquiry are relatively well-understood (hence the "U" of UNSI, as Understood). This distinguished UNSI practices from innovative practices dedicated to the investigation of newly-explored and poorly-understood phenomena. In UNSI practices, the natural phenomena under scrutiny do not pertain to a completely new domain. This excludes practices interested in phenomena hitherto experimentally undetected, the existence of which is discussed and contentious. In the practice we study, the phenomena involved have already been the object of an experimental investigation: their very existence is not questioned, and their main global features are already known. The aim of scientists is to go further in terms of precision and details.

\subsubsection{A rough mapping of scientific practices, as a basis for a typology of calibrations}

The present article focuses on calibration in scientific practices characterized by the three features (i), (ii), and (iii) - called UNSI practices. Other types of scientific practices have been involved in our attempt to delineate UNSI practices section 3.1.2 - as well as in section 2.2 although more implicitly - which do not possess these three features altogether. A systematic typology of scientific practices would be needed, because calibration arguably takes different forms in each of these other practices and in UNSI practices (see next section). Here, we shall only provide a rough mapping.

Four types of scientific practices can be contrasted with UNSI practices. Two of these types have the means of scientific inquiry as their primary target. One corresponds to professional metrological practices, which aim at establishing a standardized instrumental stratum of international scope (and which can in turn be partitioned in several, more or less innovative sub-types, see Mallard 1998). The other corresponds to the local invention of a new instrument outside of professional metrology. The latter practices, like professional metrology, struggle to increase knowledge about the means of scientific investigation; but contrary to professional metrology, they are not essentially concerned by international standardization. Typically, their aim is to achieve a particular, locally stabilized instrumental prototype. The two remaining scientific practices take natural phenomena as their 
primary target. One corresponds to the investigation of new, poorly-understood phenomena with wellmastered instruments. The other corresponds to the investigation of new, poorly-understood phenomena with poorly-mastered instruments.

\subsubsection{Situating calibration in UNSI practices with respect to calibration in different practices: a comparatively simpler and less problematic case}

For our present purpose, two points are important: first, calibration in UNSI practices differs in significant respects from calibration in the four other types of scientific practices; second, calibration in UNSI practices corresponds to one of the simplest, less problematic configuration. A full demonstration of these claims would require a complete characterization of calibration in the different types of practices involved, and a systematic comparison between them. Such 'demonstration-of-walking-by-walking' is obviously not an available option, given the limited scope of a paper. However, the two point just-mentioned seem rather obvious, at least from certain angles of comparisons. Let us give indications about the angle of comparison with respect to which they are the most obvious.

According to the second feature of UNSI practices (see section 3.1.2, (ii)), calibration in such practices is calibration from the standpoint of scientific practitioners who are users of already standardized, theoretically well-understood and practically well-mastered instrumental devices. Focusing on this feature, it is clear that calibration in UNSI practices is notably different from calibration in practices which intend to design new instruments or to investigate the natural world with poorly-mastered instruments. In UNSI practices, the instrumental piece taken as the target of calibration is already socially stabilized: many things about this target are already established, shared and taken for granted (the 'things' in question will be specified section 4, especially 4.2). Consequently, to calibrate in UNSI practices will have to do with controlling the conformity of a given instrument with what is already collectively taken-for-granted about this type of instrument. Whereas to calibrate in professional metrological practices which aim to establish a new instrumental 'universal' standard, or to calibrate in practices dedicated to the local stabilization of a new instrumental prototype, or to calibrate in practices devoted to the exploration of natural phenomena with poorly-mastered instruments, whatever it might mean in details, will have to do with the invention and collective acceptation of an instrumental novelty.

Consequently, calibration in UNSI practices will be less creative, more predetermined, more pre-codified, and hence potentially less problematic and less controversial, than calibration in practices involving new instrumental standards or poorly-mastered instrumental devices. Note, however, that this does not imply that calibration in UNSI practices is a completely straightforward matter that amounts to automatic routines and does not require ingenuity.

Finally, let us make one important last remark about the relation between calibration in UNSI practices and calibration in professional metrological practices. Although calibration in professional metrological practices will be left aside in the remainder of this article, whereas calibration in UNSI practices will be at the center, it is important to keep in mind, all along the path, the essential dependence of the first with respect to the second. Calibration activities in UNSI practices use, and most of the time take for granted, the standards established by professional metrologists. Thus the metrological calibrations come first, both logically and chronologically. Calibration in UNSI practices presupposes metrological calibration: the former takes the results of the latter (standards of all sorts) as already instituted and unproblematic. These remarks point to the relative and recursive structure of calibration activities. Calibration activities in UNSI practices exhibit a relative and recursive structure, which ultimately depends on the work of measurement experts (professional metrologists) who define and build the primary standards. Even if practitioners ignore the primary standards, and more generally ignore a large part of the metrological chain in which the instruments they use in their current scientific activities are inserted, in concreto, metrological calibration constitutes the background of calibration in UNSI practices.

\subsection{Strategic decisions}

When we scrutinize scientific practices with the aim of understanding calibration, it appears - as illustrated above, see section 1.3.1 and section 2 - that activities candidate to calibration are diversified and at first sight not obviously homogeneous. In such a situation, partly conventional decisions have inevitably to be taken, which are both conceptual and terminological: they simultaneously institute a certain cutting of the object under scrutiny (calibration) and posit some linguistic conventions (decision to name this calibration and not that). Such decisions can be motivated to a certain extent, but they are not universally compelling decisions. The intuitions can vary from an analyst to one another, and even one and the same analyst might be subject to deep and evolving hesitations (we had multiple occasions to experience this in the course of our collective research on calibration). To cope with such a situation, we have adopted a number of strategic principles which have guided several inaugural constitutive decisions. One of these constitutive decisions has already been mentioned in the previous development, namely, the decision to distinguish types of calibration depending on types of scientific 
practices. In what follows, we present some other decisions, in relation to the strategic principles we have adopted.

\subsubsection{From the simple to the complex}

To find a path in the complicated and dense jungle of activities at first glance candidate to calibration, we followed a first usual strategic principle: go from simplest cases to more and more complex cases. In other words, we first attempted to identify and characterize relatively simple isolable configurations, and then, informed by the previous investigation and armed by the tools elaborated in the process, we turned to more complicated and more problematic configurations.

This is such guiding principle which led us, after having mapped the territory of calibration into different areas calibrations in the five types of practices distinguished section 3.1.3 -, to start with calibration in UNSI practices, as the first step of our inquiry on calibration. Given the general features described in section 3.1.2, calibration in UNSI practices predictably corresponded to one of the simplest and less problematic configuration we can find.

\subsubsection{The strategy of the simple exemplar}

This is however not the end of the story, because even focusing restrictively on UNSI practices, still, multiple, not always homogeneous activities are identified with calibration by scientists (see section 1.3. for an example) and/or by philosophers, sociologists and historians of science.

To cope with such a situation, we have followed a strategy that might be called the "strategy of the simple exemplar". The strategic maxim is the following: as the first step of the investigation, construct a simple exemplar of calibration, and then, in the next steps, use the simple exemplar as an analytic tool. The construction of the simple exemplar relies on the four-questions frame (see 3.1). The final product - the simple exemplar as a result - provides determined answers to the questions of the target, the presuppositions, the aim and the procedure of calibration. Thereby, the simple exemplar offers a first rough grasp of what calibration in UNSI practices is or can be.

Let us clarify further the status and the role of the simple exemplar of calibration. The simple exemplar is an exemplar in a Kuhnian sense, which means that it has the value of a striking, often encountered, prototypical configuration (it is a "paradigm" in Kuhn's narrow sense of the term - the large sense corresponding to the disciplinary matrix). Moreover, the content of the exemplar which constitutes the starting point of the inquiry must be simple - that is, sufficiently simple to offer a telling scheme able to manifest salient features of what is at stake - without any pretention to exhaust the topic. So the simple exemplar that will be elaborated in part 4 does not pretend, neither to be sufficient to characterize the diversity of practices candidate to calibration in UNSI practices, nor to provide a set of necessary and sufficient conditions for identifying an activity of UNSI practices with calibration. As any Kuhnian exemplar, the simple exemplar is nothing more, but nothing less, than a reference point which works as a compass and an analyzer. The simple exemplar works as a compass and an analyzer, since the it helps to discuss other, less simple and more problematic candidates to calibration, the latter being analyzed in reference and by contrast to the simple exemplar. Part 5 will give substance to the previous claims and will clarify their content further, through the consideration of some activities of calibration involved in X-ray experiments. In the conclusion of this paper, we will come back in a more systematic way on the kind of work that the simple exemplar is expected to perform (section 6.2).

\subsubsection{The strategy, in brief}

To recap, our overall strategy is: to start with the most simple kind of scientific practice (UNSI practices); then to construct, for this kind of practice, a simple exemplar of calibration which offers a first schematic grasp of the kind of activity under scrutiny (in this respect, the simple exemplar is part of our characterization of the content of calibration); subsequently, to exploit the simple exemplar as an analyzer for the discussion of more complex, more problematic and possibly less prototypical candidates to calibration (with respect to this function, the simple exemplar is part of our analytical framework).

\subsection{Tools for the analysis of instrumental devices taken as the target of calibration}

In our attempts to give precise answers to the four questions of the target, the presuppositions, the aim and the procedure of calibration in UNSI practices, it appeared that conceptual distinctions about the instrumental devices taken as the target of calibration were needed. So first of all, let us introduce the distinctions we finally retained (mentioned in bold in the text below). The developments will be illustrated by means of the example of an equal-arm balance such as the one of figure 1. 
Fig. 1: A balance (a weighing scale with beam arms of equal length).

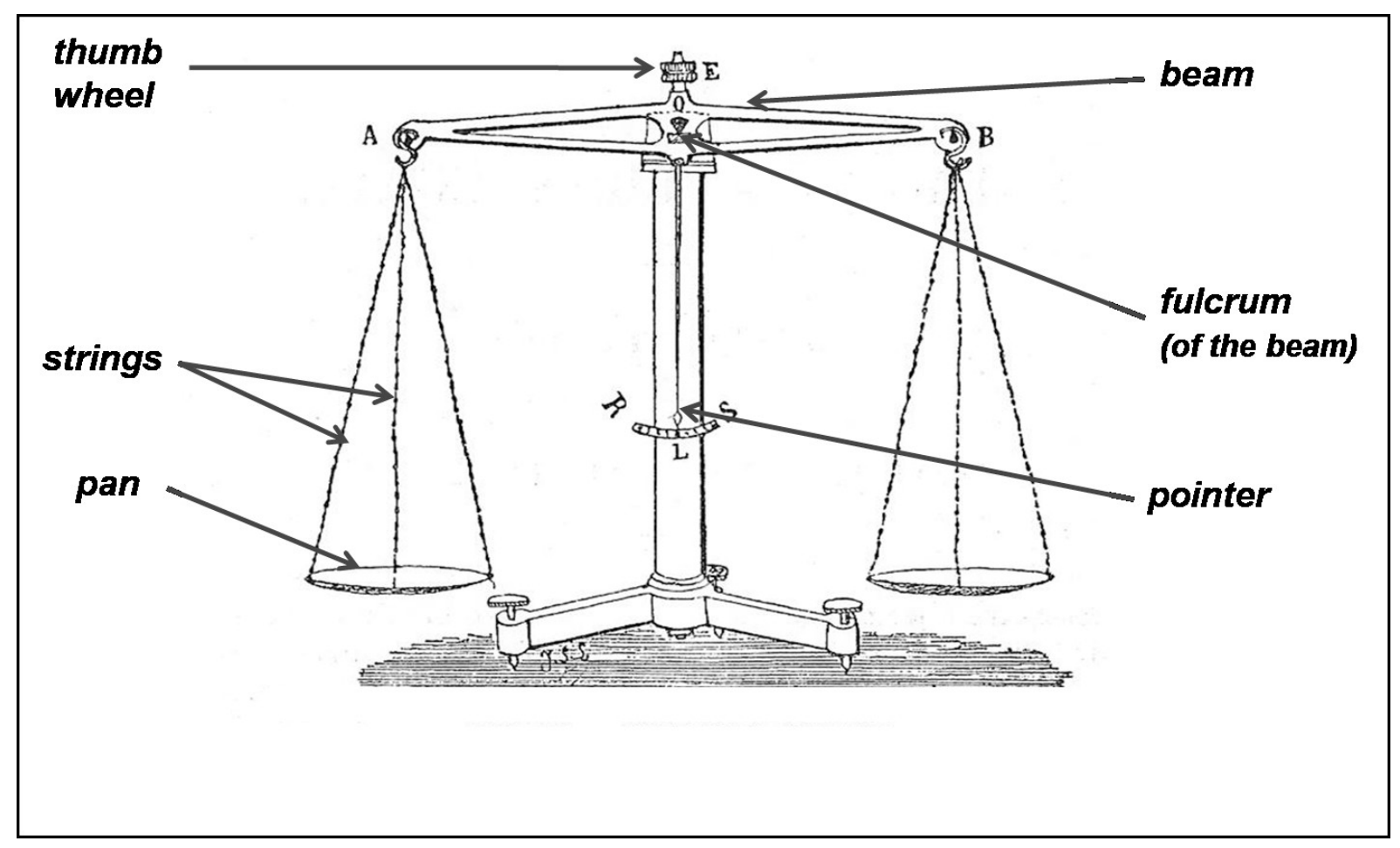

For short, we call a measuring instrumental device a 'measurer'. For example, a balance is a measurer, whereas an X-ray source is not a measurer. The function of a measurer is to evaluate quantities of a certain kind. In the case of the balance, the corresponding kind of quantity is "mass". The result of the evaluation of a quantity with a measurer usually takes the form of a numerical value associated with a measurement unit and a specified uncertainty.

\subsubsection{Types and tokens of instruments}

Two dimensions of any instrumental device need to be distinguished for the characterization of calibration, namely the type and the token.

The classical type-token distinction is actually applicable to any kind of reality, far beyond the particular case of instrumental devices. But applied to a measurer, this leads to distinguish what we call the measurer-type and the measurer-token. The measurer-type is a conceptual object, a certain kind of instrumental device. For example: a scale of the type 'balance'. The measurer-token is a particular instantiation of a given type. In our case, since we concentrate on material instrumental devices, the measurer-token is a singular material object: this particular balance here and there. For example, one of the precision balances constructed by Fortin for Lavoisier.

\subsubsection{The measurandum and the instrumental outputs}

When practitioners use a measurer-token of a certain type (for example of the type 'balance'), they want to evaluate with a certain precision, with respect to certain aims, the values of certain kinds of quantities (for example mass) in some particular conditions.

What practitioners want to evaluate in a particular measurement sequence involving a particular measurer-token, we call it the "measurandum". The measurandum corresponds to what practitioners intend to measure. The specification of the measurandum minimally requires the specification of the kind of quantity intended to be quantified in the measurements under interest (in our example, mass). But most of the time, the specification of the measurandum moreover involves the specification of multiple other elements, such as the targeted degree of precision, or some environmental conditions. For instance, if the aim is to determine the length of metallic objects, the measurandum will include the mention of the external temperature.

The measurandum must be distinguished from what we call the "instrumental outputs". The instrumental outputs refer to the measurement results considered at the 'less interpreted' level. In the case of our balance, the 
instrumental outputs would correspond to the different numbers inscribed on the different standard masses used when determining the mass of a given object, and the position of the pointer in this configuration. Beyond the particular case of the balance, instrumental outputs might be pointer deviations, numbers inscribed on digital counters, graphs... In a word, the instrumental outputs correspond to measurement results as they would be described by the layman, that is, by someone who has no idea, or a very vague idea, of what is and how works the measurer involved, of what is the aim of the measurement, etc.

When performing a measurement with a measurer-token of a certain type, strictly speaking, we do not directly obtain the measurandum. We obtain the instrumental outputs, which are then convertible, more or less directly, in the numerical value of some quantities of certain kinds (in the case of the balance, the conversion of the instrumental outputs in the mass of the weighted object is rather direct and simple).

\subsubsection{The measurer, as a means to convert humanly performed operations into the value of a measurandum through a scientific scenario based on a fundamental scientific principle}

As a result of the previous analysis, a measurer can be viewed as a means to convert certain humanly performed operations into a definite value of a measurandum. In our example, these operations are, typically: to place a material object $\mathrm{O}$ on one pan of the balance; then to add standard masses on the other pan until the equilibrium is restored. The conversion of such operations into one value of the mass of object $\mathrm{O}$ involves some scientific theories (in our case: elements of mechanics). More exactly, the passage from these operations to a value of the mass is achieved through a certain scientific scenario based on some fundamental scientific principles.

In the example of our equal-arm balance, this scenario, roughly characterized, goes as follows. When a massive object $\mathrm{O}$ is placed on one pan, it exerts on the end of one arm of the beam a vertical force $\mathrm{F}_{1}$ whose magnitude is proportional to the mass of $\mathrm{O}$. This produces a determined displacement of the beam. In order to restore the equilibrium position of the beam, standard masses are then placed on the other pan of the balance. They exert a vertical force $\mathrm{F}_{2}$, proportional to the total mass of the standards used, on the end of this other arm of the beam. If the two arms of the beam are equal, the equilibrium horizontal position of the beam is restored when the magnitude of $F_{2}$ is equal to the magnitude of $F_{1}$. The measurandum, that is, the mass of the object $O$, is then determinable from the instrumental outputs, namely, here, from the numbers inscribed on the different standard masses that have been put on the second pan in order to restore the equilibrium and from the remaining slight deviation of the pointer from the zero position on the graduated scale.

The fundamental scientific principle involved in the previous explanatory scientific scenario is often called the "lever principle". More technically, it is the principle according to which the static equilibrium of rotation is realized when the sum of the moments is zero.

\subsubsection{A scale of types: from the generic type to the model of a measurer}

A fundamental scientific principle such as the lever principle is - beyond the particular example of the balance actually what defines, at the most fundamental level, a generic type of measurer. It is what individuates a measurer as one determined generic type different from another generic type. For example what individuates the generic type 'balance' and differentiates it from another type of weighing instrument such as, for instance, the generic type 'electronic spring scale' (which characterizes, say, a digital kitchen scale).

Given the fundamental principle that defines a generic type of measurer at the most general level, a multiplicity of different sub-types can be conceived and realized (this is typically the task of engineers). For example, the lever principle is involved, and differently exploited, in a multitude of different sub-types of measurers that can all be used to assess the mass of objects (each having some specific advantages and disadvantages). A first subtype corresponds to our example, that is, an equal-arm balance. A second one is the steelyard balance (or Roman balance). A third one is the "Roberval" balance. And so on... We can conceptualize families of instruments through an indefinite multiplicity of hierarchical levels of sub-types, sub-sub-types, etc. From the highest level of the generic type, to lower levels, the measuranda, and the scenario through which one describes how the measurer converts human operations into determined values of measuranda, are defined through more and more specified descriptions which use more and more specific technical categories.

For our present purpose, we shall focus on the 'lowest-level' of such a scale of types. We call the description of a measurer at this lowest-level the model of the measurer-type (or, for short, the measurer-model).

\subsubsection{The measurer-model; optimal working and predicted deviations}

We use the term "model" as it is used when we speak, for example, of the "Ford Model T" (generally regarded as the first affordable model of automobile). On the one hand, the measurer-model is a particular sub-type of the generic type, and hence a conceptual object. But on the other hand, the description of a measurer-model intends to be the description of a real material object. It is a detailed description of a real measurer-token and its 
performances, not the characterization of an idealized measurer. It is situated at a level which is, so-to-speak, the most 'contiguous' to the concrete, material measurer.

As an illustration, in the case of an equal-arm balance, the model specifies, in addition to one version or another of the scenario sketched section $3.3 .3^{10}$ : the materials, the form and the dimensions of the beam, of the strings, and of the pans, etc.; the attainable performances, in particular the sensitivity, the maximum capacity of the balance, etc.; the conditions of use, taking into account real-life environmental variations (for example, air currents in the room can affect the balance's operation).

With respect to an analysis of calibration, it is useful to distinguish two aspects within the characterization of the measurer-model: (i) the optimal working; and (ii) the predictable deviations with respect to the optimal working.

(i) The characterization of the optimal working, including an associated uncertainty of measurement, tells the users what is at best actually obtainable from a measurer-token generated according to the model. "At best" means: just after the manufacturing of the measurer-token, assuming no defaults of fabrication, assuming normal conditions of utilization, etc. In brief, assuming the conformity of the real token to the specifications of the conceptual model.

(ii) The predictable deviations with respect to the optimal working are, for example in the case of our balance, a possible drift of the standard masses daily used, or a possible blunting of the central knife-edge (such a blunting would increase the frictions on the balancing of the beam and would then lead to a decrease of sensibility).

On the whole, the specifications of a measurer-model delimitate the identity of the model, and at the same time define what should be the identity of each individual measurer-token generated according to the model. So the measurer-model provides the normative identity of an (indefinitely large) class of individual measurer-token supposed to be instantiations of this model. It gives a normative characterization of any individual instantiation of the class defined by the model.

\subsubsection{A frame for the conceptualization of instrumental devices}

Table 1 offers a synthetic overview of the conceptual distinctions introduced section 3.3., applied to the particular case of the equal-arm balance.

Table 1: The instrument-frame (bold items), applied to the particular case of the equal-arm balance

\begin{tabular}{|l|l|l|}
\hline \multirow{4}{*}{$\begin{array}{l}\text { Generic } \\
\text { type }\end{array}$} & Generic name & Balance \\
\cline { 2 - 3 } & $\begin{array}{l}\text { Measuranda for the measurement of } \\
\text { which the generic type is designed }\end{array}$ & $\begin{array}{l}\text { Mass of certain kinds of material objects in a given } \\
\text { context with a certain degree of precision }\end{array}$ \\
\cline { 2 - 3 } & Fundamental scientific principle & $\begin{array}{l}\text { Lever principle (static equilibrium of rotation realized } \\
\text { for null sum of the moments) }\end{array}$ \\
\cline { 2 - 3 } & $\begin{array}{l}\text { Kinds of quantities typically involved } \\
\text { in the mesuranda }\end{array}$ & Mass \\
\hline \multirow{4}{*}{ Model } & Name and description & Equal-arm balance \\
\cline { 2 - 3 } & Scientific scenario & See section 3.3.3 \\
\cline { 2 - 3 } & Instrumental outputs & $\begin{array}{l}\text { Numbers inscribed on the standard masses of the } \\
\text { residual deviation of the pointer from graduation zero }\end{array}$ \\
\cline { 2 - 3 } & Predicted deviations with respect to & Drift of the standard masses used \\
\hline
\end{tabular}

${ }^{10}$ The scenario provided section 3.3.3 has been introduced at a point of the developments where the notion of model was still not available, but it should be clear from now that it is situated at the level of the model. It corresponds to one possible description of one particular model - the model 'equal-arm balance' - of the type 'balance'. The scenario is obviously too specific to be associated with the level of the generic type. What holds for the generic type (definition of the measuranda, description of the scientific scenario) must also hold for all the multiple particular models, and is then differently specified for each particular model. But the scenario proposed above does not apply to any model of the generic type 'balance'. For instance, it is not valid as a description of a balance of the model 'Roman balance' (which involves only one pan). 


\begin{tabular}{|l|l|l|}
\hline the optimal working & $\begin{array}{l}\text { Blunting of the central knife-edge } \\
\ldots\end{array}$ \\
\hline
\end{tabular}

Table 1 is intended to achieve simultaneously two aims. On the one side, it provides a substantial characterization of a particular measurer, namely of the model of balance that will be used in part 4 as the central example for the sake of the illustration of the simple exemplar of calibration. On the other side, it provides a tool of general scope, namely a general frame for the conceptualization of any measurer-token. The frame corresponds to the bold items of the Table. This frame can be applied to any newly-encountered case of measurer (see section 5, Table 3). It is conceived as a basic starting point, intended to be, when needed, either completed, or accommodated (see section 5, Table 4 for an adaptation to a non measuring devices). To the extent that this frame serves as the basis of an adaptation to instrumental devices that are not measurers, its fruitfulness is not restricted to the case of measurers, and we can thus see it as a frame for the conceptualization of instrumental devices in general. So we call it the instrument-frame.

We are now in a position to construct the simple exemplar of calibration, by providing determined answers to the questions of our four-questions frame and by using the instrument-frame.

\section{Constructing the simple exemplar by means of the four-question frame and the instrument-frame}

\subsection{Investigation of the first question: the target $T$ of calibration}

The first question is: what kind of thing can be the object of a calibration?

For the simple exemplar as we define it, the target is a measurer. To go further, we need the distinction, introduced above section 3.3.1, between the measurer-type and the measurer-token. Armed with this distinction, our answer to the first question is: for UNSI practices, and as we define the simple exemplar, the target $\mathbf{T}$ of calibration is a measurer-token.

But not all procedures directed toward a measurer-token are calibrations. So we must specify further what distinguishes a calibration test from other possible tests of a measurer-token that would not count as calibrations. One important difference lies in some presuppositions about the measurer. This leads us to our second question.

\subsection{Investigation of the second question: the presuppositions Ps of calibration}

The question is: what presuppositions Ps are constitutive of the kind of activity calibration is?

Calibration in UNSI practices involves some presuppositions about the target of calibration, that is, about the measurer. At this level, the question is: what is taken for granted about the measurer, which delimitates what is not granted and has to be checked and controlled in a calibration procedure? Our answer is: two different presuppositions about the measurer are involved in calibrations in UNSI practices.

The first presupposition is: the measurer as a conceived object is not problematic. This means that neither the generic-type, nor the model of the measurer-token, are questioned. In other words, all the elements involved in the characterization of the model, including the elements related to the generic-type, are taken to be solid, are viewed as uncontroversial pieces of human knowledge. This is clearly the case for an equal-arm balance. From a theoretical point of view, such a model of balance is a fully-understood object. Nobody questions the validity of the deeply-entrenched lever principle which defines the generic-type. Nobody contests the ability of balances of this model to enable reliable measurements of mass. So the first presupposition (say P1) is: the measurermodel is unproblematic.

Obviously, this first presupposition derives from our initial choice to concentrate on calibration in UNSI practices, that is, in practices which use already standardized, widely and for-a-long-time used, instruments. Or more exactly, to make P1 explicit and to explain, with the help of the concepts of the instrument-frame, what P1 exactly means, is a way to explicate important aspects of what is meant when we say that UNSI practices use standardized instruments: standardized measurers are unproblematic measurers in the sense explicated in the previous paragraph.

The second presupposition (say P2) is: the measurer-token is not defective. In other words, there is no breakdown, no failure. Globally, the measurer works properly - even if it is not necessarily precisely adjusted. Re-described in our categories, the distance between the measurer-token and the measurer-model is not too important. Obviously, this assumption can be questioned in the course of a sequence of actions first conceived as a calibration. But as soon as this assumption is abandoned, the nature of the activity changes, and the activity 
then involved is, according to our conceptual and terminological decisions, no more adequately categorized as $a$ calibration. If practitioners conclude that the measurer-token is defective, typically, the operations that follow correspond to a repair, not to a calibration.

\subsection{Investigation of the third question: the aim of calibration}

Our next and third question is: what is the aim of a calibration applied to a measurer-token $\mathrm{T}$ under presuppositions Ps? Our answer will distinguish the proximate aim and some more distant aims ${ }^{11}$.

\subsubsection{The proximate aim of calibration}

Expressed at the more general level, the proximate aim of calibration is to master the possible gap between the measurer-token and the measurer-model. More precisely, the aim is to master the distance between:

On the one hand, the instrumental outputs actually obtained with this individual measurer-token at a given time in a given context (in our example: the different numbers inscribed on the different standard masses used to restore the balance equilibrium and the residual deviation of the pointer, which are convertible in the value of a certain measurandum, here the mass of an object);

On the other hand, the value of the mesurandum that should have been obtained in the optimal configuration (that is: if this measurer-token as used in this context actually coincided with the measurer-model in optimal working).

For short, we will call the difference between these two items the obtained / optimal discrepancy. So in normal practices of nature investigation, the aim of calibration is to master the obtained / optimal discrepancy for a measurer-token at a given moment in a given context, assuming P1 and P2. This is what we call the proximate aim of the activity of calibration, that is, the most immediate aim.

\subsubsection{The distant aims of calibration}

Beyond the proximate aim, calibration also serves other, more distant aims, which could be brought into the picture, in the perspective of a more complete and meaningful characterization of calibration. As a crucial illustration, we can stress that a more distant aim of any calibration is to create the conditions for, and to actually obtain, measurement values that are commensurable with the international system of units. Obtaining this commensurability is a condition for another distant aim, namely, to ensure a universal (international) confidence in measurements performed all over the world in different places and conditions.

Although it would be desirable to introduce such distant aims of calibration into the picture, calibration in UNSI practices is nevertheless understandable independently of the specification of most of its multiple possible distant aims. However, beside the proximate aim, there is one distant aim that cannot be left aside for the understanding of calibration in UNSI practices. It cannot be left aside, because it impacts the way the proximate aim of calibration is specified and translated into a concrete procedure in practice. This distant aim is to obtain reliable results in subsequent, more or less pre-determined targeted measurements. We call the latter measurements the end-measurements.

\subsubsection{Reliable end-measurements as the essential distant aim of calibration in UNSI practices}

The expression "end-measurements" intends to play on the two-fold meaning of the term "end". First, it intends to suggest the idea of finality as involved in a means-end relation: here, the end is to perform reliable subsequent measurements, and calibration is one means with respect to this aim. Second, it intends to suggest the idea of the end of a story: in our case, the end of an experimental sequence in which calibration plays the role of the introductive chapter.

A procedure of calibration in UNSI practices is never accomplished just for itself, but always with the intention to perform some end-measurements. So a calibration procedure in UNSI practices cannot be adequately thought in isolation, independently of its relation to the end-measurements, because the calibration of one and the same measurer-token may vary, depending on the purpose of the end-measurements.

\subsubsection{Specification of the proximate aim taking into account the end-measurements}

We can inject the constraints associated to the end-measurements into the definition of the proximate aim. The proximate aim of calibration would then be: to master the obtained/optimal discrepancy that matters given the measurandum of the end measurements.

\footnotetext{
${ }^{11}$ This terminology is borrowed from Hasok Chang's framework for activity-based analysis (Chang 2013). The proximate aim is also called by Chang the "inherent aim".
} 
Suppose, for example, that the end-measurements intend to measure, with a precision of +-1 gram, the mass of an object $\mathrm{O}$, and that this mass is expected to fall within an interval of 20 to 40 grams. The calibration procedure of the same balance-token will not be the same as if the end-measurements intended to measure, with a precision of +-1 grams, another object for which the mass is expected to fall within an interval of 200 to 400 grams. As the obtained/optimal discrepancy is not necessarily the same across the range of the balance, the calibration procedure must attempt to determine this discrepancy for an interval of values which is relevant regarding the specific range of values that will be involved in the end-measurements.

More generally, the calibration procedure must master the obtained/optimal discrepancy for a range of values, a degree of precision, etc., which are relevant with respect to the ones involved in the end-measurements. Thus, the most precise formulation of the proximate aim of calibration in UNSI practices is: to master the obtained / optimal discrepancy for a measurandum which fits with (is as close as possible to) the measurandum of the end measurements.

\subsection{Investigation of the fourth question: the procedure of calibration}

Our fourth and last question is: through what kind of procedure is the aim of calibration achieved? What are the structural elements and logical steps of a calibration procedure?

In section 4.3, we wrote that the aim was to master the obtained / optimal discrepancy. Now we have to specify what the verb "to master" means here. It means: first, to determine the discrepancy (which implies most of the time to evaluate the gap quantitatively); second, if needed - depending on the result of the previous evaluation and on the characteristics of the end-measurements - to correct the discrepancy or to take it into account in a way or another. So a calibration procedure can be divided into two logical moments: first a moment devoted to calibration tests; second, a moment consisting in the application of calibration operations according to the conclusions of the testing stage.

\subsubsection{Calibration tests}

Concerning calibration tests, two species are commonly involved in UNSI practices: blank calibration tests and calibration tests with a measurement standard.

\section{a/ Blank calibration tests}

Blank (or background) calibration tests give the blank indication of the device under test. For example, the blank calibration of our balance would consist in assessing the position of the balance pointer when the pans are empty.

The calibration test is 'blank', in the sense that a measure is undertaken in the absence of any object of the same kind as the object the end-measurements aim to characterize (note here one more time the indispensable reference to the end-measurements).

The principle of a blank calibration test is to perform measurements in all points similar to the endmeasurements (same measurer-token, same experimental context, same kinds of measured quantities, etc.) except one: no measured object of the kind involved in the end-measurements is involved in the calibration tests (in our example: no material object is placed on the pans of the balance). Ideally, the blank calibration test measurement and the end-measurements are in the following relation: without/with a measured object of the kind under study all other things being equal. If this is actually the case, the blank test gives the background noise, specifically due to the measurer-token, that will be present in the end-measurements.

This noisy contribution of the measurer-token is determined by a comparison between the instrumental outputs obtained as the result of the blank calibration (for example: a position of the pointer corresponding to 1 gram), and the prescriptions of the model in optimal working (i.e., a position of the pointer corresponding to the equilibrium zero position, taking into account the precision of the reading). The difference, that is, the signal generated by the balance-token in the absence of any material object placed on the pans, is something that practitioners must be careful not to count as a contribution of the object under study.

\section{b/ Calibration tests with etalons}

A measurement standard (in French: "etalon”) is an object of already well-known properties. It can be a metrologically certified measurement standard, or, if not certified, an object with sufficiently well-known and stable properties, which is used by practitioners as a working measurement standard. Since the English word "standard" has a very broad sense which might create ambiguities in the context of our discussion, we prefer to use the French word "etalon". So the second species of calibration test corresponds to calibration tests with etalons. Franklin's and the VIM's definitions of calibration, discussed section 2.2, correspond to calibration tests with etalons. 
Calibration tests with etalons consist in measuring on the etalon, by means of the measurer-token under test, quantities of already-known values. In the example of the balance, the etalons correspond to certified etalon masses, or at least, to etalon masses the values of which have been previously determined more precisely than are determined the values of the standard masses daily-used as part of the balance-token under test. A calibration test with etalon of the balance (that is, a calibration test of the balancing mechanism plus the daily-used standard masses), would be: to place an etalon on one pan of the balance; then to place daily-used standard masses on the other pan in order to restore the equilibrium as much as possible; and, finally, to evaluate the difference between the mass actually measured in the test and the already-known mass of the etalon. Since the mass of the etalon is known in advance, the instrumental outputs that should be obtained with a measurer-token of this model if the token coincided with the optimal working are pre-determined. In our example, the position of the pointer and the different numbers inscribed on the different standard masses used to restore the balance equilibrium, which are convertible in a measured value of the mass of the etalon, should coincide with the certified value of the mass of the etalon. If the calibration test leads to a value significantly different, the difference corresponds to the obtained/optimal discrepancy (say: plus 2 grams). This contribution of 2 grams must be attributed to the measurer-token as used in this context, and not to the object under study in the end-measurements.

Ideally, a calibration test with etalon is in all points identical to the end-measurements, except in one respect: the measured-object used in the calibration test is a token with already well-characterized properties, known before and independently of the calibration test. Except in this respect, the calibration test is similar to the endmeasurements, in that the calibration test (ideally) involves: the same measurer-token; the same experimental context; a measurandum as close as possible to the measurandum of the end- measurements, and in particular, a measurandum which involves the same kind of measured-object.

This latter condition, 'same kind of measured-object', is very important. To understand why, we have to remember (see section 4.3.4) that the aim of calibration is to master the obtained / optimal discrepancy for $a$ measurandum which is as close as possible to the measurandum of the end-measurements. Applied to a calibration test with etalon, this condition imposes on the etalon to be as similar as possible to the objects under study in the end-experiments. If the etalon is too different from the object under study in the end-measurements, the information obtained as a result of the test with etalon are not applicable to the end-measurements. This point has been stressed by Harry Collins, in the context of his debate with Franklin about the power of calibration with respect to the experimenter's regress. Collins talks about "the assumption of near identity of effect between the surrogate signal and the (...) signal that is to be measured (detected) with the instrument" [in what we have called the end-measurements] (Collins 1992 [1985], 105). Franklin expresses the point as "the adequacy of the calibration, that is the 'near-enough' identity of the surrogate signal with the desired signal” (Franklin 1997, 75).

Taken all what has been developed about the etalon in this section, we can say that a calibration test with etalon in UNSI practices involves, in addition to the two presuppositions P1 and P2 about the measurer-token, two supplementary presuppositions about the etalon:

P3: the values certified or assumed about the etalon are indeed reliable (for short, say: reliability of the etalon)

P4: the etalon is sufficiently similar to the object under study in the end-measurements (for short, say: adequacy of the etalon).

\section{c/ A brief comparison between the two species of calibration tests}

The underlying logic of a calibration test with an etalon, as well as of a blank calibration test, is a differential logic in which the end-measurements play the role of a reference point. In both cases, a circumscribed contrast is created between the end-measurements and the calibration tests. In both cases, this contrast allows to delimitate differentially what must be attributed, on the one hand to a bias introduced by the measurer-token as used in this context, and on the other end to the measured-objects under interest.

The difference between the two cases lies in the fact that in calibration tests with etalons, the measurer-token is in interaction with a measured-object of the kind under interest (the measurer-token is employed in conformity to its intended function and normal use, namely, to indicate the mass of weighting objects placed on its pans). In one case or the other (with / without weighting objects on the pans), the possible obtained / optimal discrepancies are not necessarily due to the same causes. Hence the two species of calibration tests provide possibly different and complementary information about the measurer-token and its possible drift with respect to the optimal working as defined by the measurer-model.

Once calibration tests have been performed, practitioners have to decide, according to the obtained / optimal discrepancy they have found and to some desiderata imposed on the end-measurements, if calibrating operations are required or not, and, if so, which ones.

\subsubsection{Calibration operations}


Two kinds of calibrating actions are commonly involved in prototypical calibrations: material operations and symbolic operations.

Material operations correspond to concrete manipulations exerted on the individual instrument, which introduce effective and tangible modifications of the measurer-token as a material body. For example, with the balance, following the blank calibration test described in point a/ of section 4.4.1: to manipulate a thumb wheel in order to restore the equilibrium of the beam by displacing it slightly with respect to its fulcrum, so that the pointer, which initially coincided with 1 gram, finally coincides with the zero.

Symbolic operations are diversified, but as a prototypical illustration, they correspond to mathematical corrections applied to the instrumental outputs actually obtained in the end-measurements. In the case of our balance, it would for example amount, following the calibration test with etalon described in point $\mathrm{b}$ / of section 4.4.1, to subtract 2 grams to the values actually obtained in the end-measurements.

Material operations transform the measurer-token so as to make the real material instrument as close as possible to the model in optimal working. Symbolic operations do not intervene on the measurer-token as a concrete physical object: the gap between the real individual instrument and the model in optimal working remains unchanged; the discrepancy is treated intellectually, by an intellectual operation applied to the instrumental outputs actually obtained in the end-measurements.

\section{5. A synthetic overview of the simple exemplar of calibration in UNSI practices}

Table 2 recaps the main elements developed in part 4.

Table 2: The simple exemplar of calibration in UNSI practices

\section{Calibration in UNSI practices: a simple exemplar}

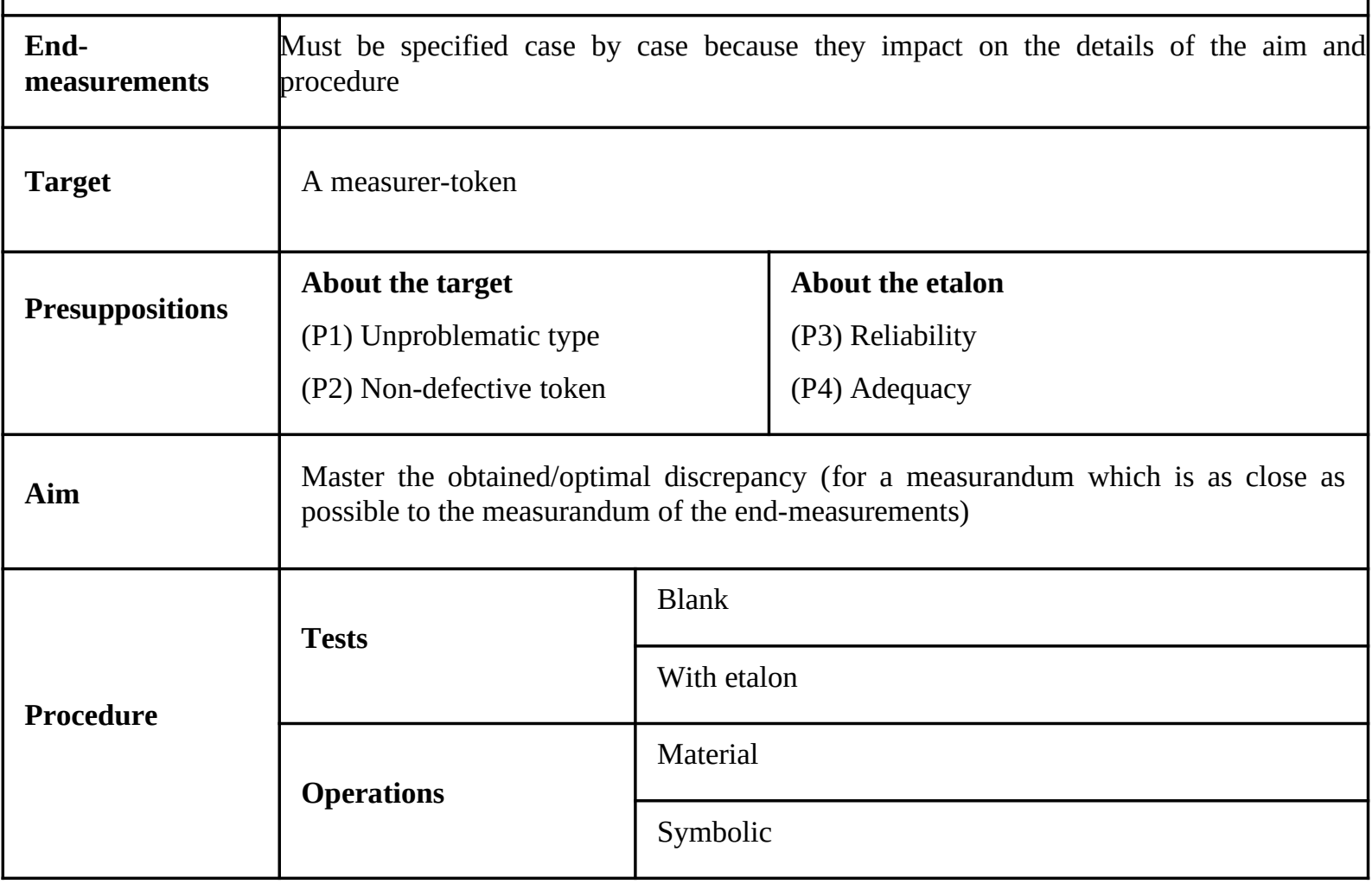

Table 2 provides a conceptual framework for the analysis of instances of calibration in UNSI practices. The framework will be used below, in part 5, for the analysis of X-ray experiments. This use will show the kind of work accomplished by the framework.

\subsection{Qualifications, complements, indications for further research}


To close our presentation of the simple exemplar, we would like to stress that the characterization given above could be refined in diverse respects.

\subsubsection{Environmental factors}

Our characterization of the simple exemplar did not sufficiently consider the issue of environmental factors. True, our characterization did not completely ignore such factors. In particular, the aim of calibration has been defined as the evaluation - and correction if needed - of the distance from its optimal working of "a measurertoken at a given moment in a given context". In this definition, the mention of "a given context" encompasses possible environmental influences. Moreover, our characterization of the model of an instrumental device also mentioned elements that are on the side of environmental variables (see section 3.3.5: "the model specifies (...) the conditions of use, taking into account real-life environmental variations"). But beyond such simple indications about the importance of environmental factors, we did not go into the details about how to take the environment into account. Such details depend to a large extent on the particular model of measurer under scrutiny, but they should be incorporated in a refined picture of calibration in UNSI practices.

Let us just say one word in this perspective. For standardized models of instruments, the specifications of the model provide information about the environmental factors amenable to influence the instrumental outputs of measurements undertaken with this instrument. When it is known that some environmental factors can affect a measurer, these environmental factors have to be controlled. In the case of the equal-arm balance, environmental conditions such as draughts, vibration, temperature changes, or air density changes can affect the balance's operation. These factors have to be assessed, particularly if a change of location of the balance has occurred. For some of these factors, typically temperature changes and air density changes, corrections can be introduced. Take, for example, air density changes. Air density varies with pressure, temperature and relative humidity. A variation of air density leads to an air buoyancy variation, which can affect the values of the masses measured with the balance-token. Hence an air buoyancy correction may be needed, by multiplying the measured-values by a correction factor. This correction factor is calculated by taking into account the density of air during the weighing (which can be estimated by a formula linking air density to air pressure, relative humidity of the air and air temperature), the density of the standard (certified) masses and the density of the material being weighed.

\subsubsection{Repeated measurements and statistical treatment for the purpose of evaluating measurement precision}

In our characterization of the simple exemplar, we have made as if the evaluation of the value of a given measurandum in the calibration tests (for example the value of the mass of a given etalon provided by a given balance-token in a calibration test with etalon) only required to perform one single measurement with the measurer-token. This is, of course, a simplification.

We have ignored, here, the practice of repeating same measurements for the evaluation of one and the same measurandum (for example, the repetition of several same weighting measurements of the same etalon with the same balance-token), and the statistical treatment of the different values obtained (calculation of the mean, of the standard deviation, etc.).

However, even if it has been completed so as to take into account this point, the simple exemplar of calibration in UNSI practices, as characterized above, would still not correspond to what might be called a 'full' or 'systematic' calibration procedure. Without going into too many details, let us nevertheless give some indications about what 'full' calibration procedures are, and what is their relation to the simple exemplar of calibration as described above.

\subsubsection{The simple exemplar of calibration in UNSI practices in relation to 'full' calibration}

A full calibration procedure of one and the same measurer-token includes multiple tests directed toward different parameters, such as the measurements repeatability, the measurements error across the range of the measurer, the sensitivity, etc. Using the results of these tests, a global uncertainty of measurement is calculated, which is assumed to hold for subsequent measurements, providing a ceteris paribus condition can be assumed to hold.

Whereas calibration as described by the simple exemplar is performed almost each time a determined sequence of end-measurements is planned, a full calibration procedure is performed less frequently. A full calibration procedure is performed when the balance enters service in the laboratory, when a significant change in the laboratory's environmental conditions occurs, when a change in location or position of the balance occurs, when daily calibration tests show a significant change with respect to what is expected according to the model and to the results of the last full calibration procedure, and in any case - even if no contingent event of the previous kind is suspected to have occurred - regularly (but not frequently: for example each year). By contrast to a full calibration procedure, our simple exemplar describes 'daily' or 'before-use' calibration procedures. Relying on the categories previously introduced, the most exact formulation would be: 'before a planned sequence of end- 
measurements'. For short, let us say 'before-end-measurements', or vaguer but shorter, 'daily' calibration procedures.

The relations between full and daily calibration tests are as follows. The information provided at the end of a full calibration procedure - in particular the resulting uncertainty of measurement - is taken to be valid for any subsequent measurements, providing that no consequential change has occurred after the full calibration procedure. Thus, this information is taken to be valid both in the subsequent daily calibration tests, and in the following end-measurements with respect to which the daily calibration tests have been undertaken. This information actually constitutes the background of the daily calibration tests as described through our simple exemplar. Moreover, it gives precise indications about some features of the results of the daily calibration tests that would show that a new full calibration should be performed. Such indications, expressed in our categories, typically correspond to a maximal allowed obtained / optimal discrepancy, which is specified as a result of the full calibration procedure. If the limit associated with this maximal allowed discrepancy proves to be exceeded in the before-end-measurements calibration tests, a full calibration should be carried out.

A full calibration procedure is "systematic" in a double sense. First, the corresponding sequence of tests is less dependent on the specificities of the planned end-measurements than the before-end-measurements calibration tests (as we have seen section 4.3.3, before-end-measurements calibration tests cannot be adequately thought independently of their relation to the end-measurements). Second, the sequence of tests must be performed regularly, even if no event has occurred (such as an accident) that leads to suspect that the measurer-token has drifted from the optimal working.

In order to indicate directions in which the simple exemplar of calibration would have to be completed, we shall now give some fragmentary insights about what may be a full calibration procedure, relying once more on the example of the equal-arm balance. As already pointed out, the full calibration procedure of a measurer-token includes a multiplicity of calibration tests directed toward different parameters which altogether characterize the quality of that measurer-token. For an equal-arm balance, a full calibration procedure includes a test of repeatability, a test of measurement error across the range of the balance, a test of sensibility, a test of eccentric loading ("off-centre loading”)... Let us say a little bit more about one of these tests, perhaps the most important one: the test which aims at evaluating (what is commonly called) the measurement error across the range of the balance-token.

\subsubsection{A calibration test of the measurement error across the range of the measurer-token, as an estimation of the accuracy: the construction of a calibration curve}

The parameter under test is the measurement error across the range of the measurer-token. It is often expressed in the form of a calibration curve. It corresponds to an estimation of the accuracy of the measurer-token across its range.

The principle of the test, illustrated with the case of the balance, goes as follows. Different etalons of increasing mass value are used, and a measurement is performed, by means of the same balance-token under test, for each of these etalons. Then, a calibration curve is constructed. Each measured value is plotted against the corresponding nominal value of each certified etalon (standard mass) ${ }^{12}$, and a curve is generated by a fit through the points.

The calibration curve provides two types of crucial information about the measurer-token. The first type concerns each point of the curve, considered one by one. For each point of the curve, given the corresponding nominal mass value of the etalon, which is known and certified, the calibration curve shows the departure of the measured-value from this nominal value - that is, what we have categorized as the obtained / optimal discrepancy. The calibration curve provides, for each point of the curve, an estimation of the difference between the mass-value that should have been obtained with the balance-token, given the maximal precision obtainable with this balance-model in optimal working, if the balance-token coincided with the optimal working, and the mass-value that is actually obtained with this balance-token applied to the etalon in this context. Thus, our description of a calibration test with an etalon in the simple exemplar corresponds to any single point of a calibration curve.

The second type of information provided by a calibration curve derives from the consideration of the curve as $a$ whole. The consideration of the curve as a whole enables an assessment of the linearity of response of the balance-token across its range. Such assessment gives crucial clues about the possible causes of the observed deviations from the optimal working - as well as about the frontier between a not well calibrated and a defective

\footnotetext{
${ }^{12}$ More exactly - since for each standard mass, several repeated same measurements are most of the time performed - the ordinates points of the curve are constructed according to the statistical procedure mentioned in section 4.6.2: for each, the mean value of the different measured values is used.
} 
balance-token. When trying to identify the potential sources of errors - the potential sources of the recorded deviations from the optimal working - a comparison of the slope of the obtained curve with the slope of the curve specified by the model for the optimal working can be performed. Suppose that the curve deviates from linearity more and more as the load increases. Practitioners will suspect, for example, a slight shift of the beam from its optimal position, with the result that the two arms of the balance can no more be considered as being of equal length.

According to the outcome of this calibration test, calibration operations may be needed. These operations can be - using the categories introduced section 4.4.2 - either material or symbolic. In the examples just given, providing a shift of the beam is suspected, a material calibration operation would be to displace the beam with respect to its fulcrum so as to reestablish the 'equal-length' condition required by the model. Symbolic calibration operations could be applied as well to the instrumental outputs of the end-measurements, in the form of mathematical corrections which, in the present case, would vary according to the range of loading.

\subsubsection{Concluding remarks on full calibration}

The previous elements should be elaborated in order to complete the characterization of calibration in UNSI practices. Our simple exemplar describes before-end-measurement calibration procedures - like checking the zero (blank calibration test) and checking the measured values obtained when an etalon is used (calibration test with etalon) - but leaves aside estimations of precision and uncertainty. Such before-end-measurements procedures are important, because they inform about a possible instrumental drift with respect to the optimal working and enable to decide what calibration operations can be performed in order to accommodate the drift. However, they do not exhaust calibration tests in UNSI practices. They are conduced given the result of a previously performed full calibration procedure.

As a conclusion of the above reflections about full calibration, let us stress that in such procedures, the trust we have in the certified etalons used is absolutely crucial, since the reliability of the conclusions of a full calibration procedure essentially depends on the certified data (nominal values and uncertainties) attached to the etalons used. This echoes to the remarks made at the end of section 3.1.4, where we stressed that calibration in UNSI practices presuppose the results of professional metrologists.

Using our simple exemplar of calibration as a framework, we are now going to investigate a more complex case of calibration in UNSI practices.

\section{A more complex case of calibration in UNSI practices: Calibration in X-ray diffraction experiments in a nanoscience Laboratory}

\subsection{General features and interest of the more complex case}

The case deals with X-ray diffraction experiments and the related calibration procedures currently undertaken at the Jean Lamour Institute (hereafter IJL) in Nancy. This is the Institute where our colleague Cathy Dufour worked as a physicist, as a member of the "Nanomagnétisme et électronique de spin" team (nanomagnetism and spin electronics). The IJL has an expertise in the growth and production of very thin (nanometric) films with specific properties. The experiments we are going to discuss concern the characterization of the internal structure of mono-crystalline thin-films by means of an X-ray diffractometer.

These experiments can be assimilated to UNSI practices: we can consider that they investigate relatively wellUnderstood Natural phenomena with Standardized Instruments. This is obvious as far as the means of the investigation is concerned: the X-ray diffractometer used at the IJL is an already standardized, widely used instrumental device. This is perhaps less obvious as far as the phenomena under inquiry are concerned, since one aim of practitioners at the IJL is to produce material thin-films with new interesting properties. To discuss seriously the issue of the 'degree of novelty' of the phenomena under inquiry would require more developments, but here, we will take the point for granted, relying of the fact that the kinds of material samples under study, namely mono-crystals, are already well-understood materials (with an available well-characterized repertory of kinds of crystalline structures, etc.).

The calibration activities we are going to study are more complex than the one analyzed in part 4 relying on the example of the balance, because they involve a more complex instrumental device, in the sense that the target of calibration is treated as an instrumental system composed of multiple parts.

Compared with the simple exemplar of calibration illustrated by means of the balance, this more complex case of calibration is peculiar and instructive in (at least) two important respects. First, the fact that it involves, as the target of calibration, a treated-as-composed instrumental system, directs the attention on the relations that might hold between calibration of an instrumental device as a whole and calibration of its parts. Second, this case 
points to instances in which the target of calibration is not a measurer, with some epistemological consequences that will be examined.

\subsection{The end-measurements}

In order to characterize the calibration procedures related to the X-ray experiments undertaken at the IJL, the first thing to do is to describe the end-measurements, since as stressed in section 4.3, different end-measurements may impact differently the details of the calibration of one and the same measurer-token.

In the particular case analyzed below, we consider two (related) kinds of end-measurements successively undertaken by practitioners. In the first ones, the aim is to determine the inter-reticular distance $d$ between the crystalline planes of the thin-films synthesized at the laboratory at a given time (say $\mathrm{t}_{1}$ ). This is informative about the growth process and about the structural quality of the thin-film sample that has been produced (is it a 'perfect' crystal? What is the degree of stress of the thin layer?). In the second kind of end-measurements, the aim is to check the stability of the sample over time (the possible evolution of the quality of the thin monocrystalline layer produced and characterized at $t_{1}$ ). This evaluation involves the reiterations of similar measurements at different moments posterior to $t_{1}$ (say $t_{2}, t_{3}$, etc.), and the creation of conditions that enable to compare (i.e., to situate on one and the same scale) the values respectively obtained $a_{1}, t_{2}, t_{3}$, etc. In what follows, we begin to analyze the situation in reference to the first kind of end-experiments. The second kind of end-measurements enters into play subsequently (from section 5.5), in relation to the calibration procedure which intends to master the intensity delivered by the X-Ray source.

Before performing end-experiments aiming at the characterization of the inter-reticular distance $d$ of a crystalline thin-film, practitioners want to test the reliability of their X-ray diffractometer-token. With respect to these endmeasurements and to the robustness of their results (the value attributed to $d$ ), the target $\mathrm{T}$ of the calibration procedure is the diffractometer-token as a whole - since the end-measurements of course use the whole diffractometer-token. So in what follows, we shall begin to analyze the diffractometer-token involved at the IJL, by means of the conceptual tools introduced so far. Then we will say a word about the calibration of this diffractometer-token as a whole, before to give a more detailed characterization of one particular step of this procedure, directed toward one particular part of the diffractometer: the $X$-ray source-token.

\subsection{The X-ray diffractometer used at the IJL: an analysis by means of the instrument- frame}

Table 3 below is obtained by applying the conceptual frame offered by Table 1 to the X-ray diffractometer-token used at the IJL. The result is a synoptic characterization of this diffractometer by means of the conceptual tools previously introduced. A number is associated to some items of Table 3: it refers to further explanations, given below, about the corresponding item.

Table 3: A conceptualization of the X-ray diffractometer-token used at the IJL

\begin{tabular}{|l|l|l|}
\hline $\begin{array}{l}\text { Generic } \\
\text { type (1) }\end{array}$ & Generic name & X-ray diffractometer \\
\cline { 2 - 3 } & $\begin{array}{l}\text { Typical measuranda for the } \\
\text { measurement of which the generic type } \\
\text { is designed (1a) }\end{array}$ & $\begin{array}{l}\text { Structural properties of crystalline material samples } \\
\text { (ex. inter-reticular distances; kind of structure...) }\end{array}$ \\
\cline { 2 - 3 } & Fundamental scientific principle (1b) & Diffraction of X-ray by a crystal; Bragg's law \\
\cline { 2 - 3 } & $\begin{array}{l}\text { Kinds of quantities typically involved } \\
\text { in the mesuranda (1c) }\end{array}$ & $\begin{array}{l}\text { Angle } \theta \text { at which a given intensity is diffracted } \\
\left.\text { Intensity of diffracted X-ray ( } \mathrm{I}_{\text {diff }}\right) \\
\text { Diffraction spectra } \mathrm{I}_{\text {diff }}(\theta)\end{array}$ \\
\hline $\begin{array}{l}\text { Model } \\
\text { (2) }\end{array}$ & Name and description (2a) & $\begin{array}{l}\text { X'pert Pro MRD PANalytical } \\
\text { Four-circle diffractometer } \\
\text { High resolution X-ray diffractometer for } \text { thin-film } \\
\text { analysis } \\
\text { Components: see the list below }\end{array}$ \\
\cline { 2 - 3 } & Scientific scenario (2b) & See description below \\
\hline
\end{tabular}




\begin{tabular}{|l|l|l|}
\hline Instrumental outputs (2c) & Graphs convertible in diffraction spectra $\mathrm{I}_{\text {diff }}(\theta)$ \\
\cline { 2 - 3 } & $\begin{array}{l}\text { Predictable deviations with respect to } \\
\text { the optimal working (2d) }\end{array}$ & $\begin{array}{l}\text { Decrease of the intensity of the X-Ray beam over } \\
\text { time }\end{array}$ \\
\hline
\end{tabular}

\section{(1) About the generic type of the $\mathrm{X}$-ray diffractometer}

(1a) The function of an X-ray-diffractometer is the determination of some structural properties of crystalline material samples, for example the inter-reticular distances between crystalline planes, the kind of crystalline structure (cubic etc.) or the like. These are the kind of properties for the measurement of which the generic type have been designed, and the kind of properties that practitioners typically intend to measure (i.e., typical measuranda) when they use an X-ray-diffractometer.

(1b) The fundamental scientific principle that defines the generic type is the phenomenon of diffraction of Xray by a crystal. The phenomenon of diffraction is the result of a particular type of interaction between radiation and matter. An X-ray beam is conceived as a certain kind of radiation constituted of photons (the range of X-ray wavelengths is placed between the ultraviolet region and the region of $\gamma$-rays emitted by radioactive substances). When an X-ray beam is directed on a crystal, different physical interactions occur. In particular, some photons of the incident X-ray beam interact with the electronic clouds of the atoms of the crystal and are deflected without a loss of energy. These deflected photons constitute the scattered radiation. The scattered radiation presents certain characteristic properties which depend on the internal structure of the crystal. More precisely, some properties of the scattered X-rays (the direction and the intensity of the scattered beam, called the angle of diffraction and the diffracted intensity) are connected to some properties of the internal structure of the crystal (such as, for example, the inter-reticular distance $d$ between crystalline planes), through determined and well-known laws. These laws are, for the X-ray diffractometer, the equivalent of the lever principle for the balance. They correspond to the fundamental scientific principle which underlies and define the type of measurer that an X-ray diffractometer is.

In the case of end-measurements aiming at the determination of the inter-reticular distance $d$ between crystalline planes, the relevant law is the fundamental law known as the "Bragg's law":

$2 d \sin \theta=n \lambda$

$d$ is the inter-reticular distance for one stacking of planes

$\theta$ is Bragg's angle, that is, the angle between the incident X-ray beam and the reticular planes

$n$ is an integer

$\lambda$ is the wavelength of the monochromatic $\mathrm{X}$-rays beam (the interval of $\lambda$ of particular usefulness in crystallography ranges between 0.4 and $2.5 \AA$ ).

The Bragg's law relates $d$, the quantity intended to be determined, to a measured-quantity, namely the angle at which the X-ray beam is diffracted and collected (see figure 2).

Fig. 2: Bragg diffraction from a cubic crystal lattice. Plane waves incident on a crystal lattice at angle $\theta$ are partially reflected by successive parallel crystal planes of spacing $d$. The superposed reflected waves interfere constructively if the Bragg condition $2 d \sin \theta=n \lambda$ is satisfied. [Title and image reproduced from $<$ https://commons.wikimedia.org/wiki/File:Bragg_diffraction.png>; GNU General Public License] 


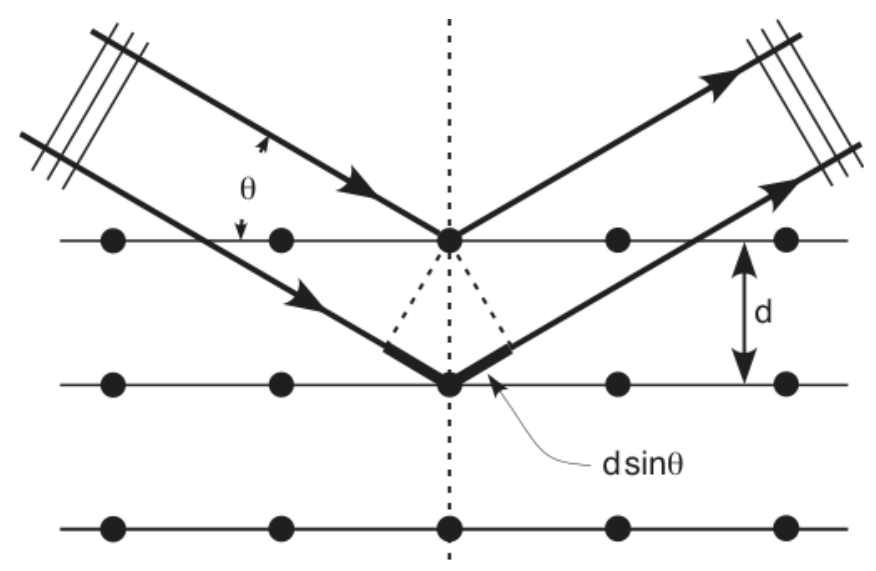

Relying on figure 2, it appears that as far as scientists are able to generate an X-ray monochromatic beam of a certain known intensity $\mathrm{I}_{\text {inc }}$, to direct the incident intensity $\mathrm{I}_{\text {inc }}$ on a crystalline sample according to a certain known angle $\theta$, and to record the intensity $\mathrm{I}_{\text {diff }}$ of the $\mathrm{X}$-rays diffracted by the sample at an angle $2 \theta$, scientists are able to determine the inter-reticular distance $d$ between the crystalline planes. This is precisely what a diffractometer accomplishes: a diffractometer is a means to convert the diffracted intensity as a function of the angle $\left(\mathrm{I}_{\text {diff }}(\theta)\right)$ into structural properties of a crystal.

(1c) It follows from what has just been said that the instrumental outputs of an X-ray diffractometer must be interpretable in terms of diffracted intensities and angles of diffraction. Thus, the kinds of quantities typically involved in the mesuranda associated to measurements with a diffractometer are angles and intensities of $\mathrm{X}$ rays. More precisely, the determination of the measurandum is achieved through a determination of the angles at which an intensity is diffracted, that is, through the determination of the different $I_{\text {diff }}(\theta)$ s. This is what is called a diffraction spectra $I_{\text {diff }}(\theta)$. From such diffraction spectra, practitioners can go back, through Bragg's law, to the values of the mesurandum under interest, in our case, to the values of the inter-reticular distance $d$.

\section{(2) About the model of the $\mathrm{X}$-ray diffractometer}

(2a) The model of the diffractometer-token currently used at the IJL is sold under the technical name "X'pert Pro MRD PANalytical” (PANalytical is the trademark). Figure 3 shows a picture of the corresponding diffractometer-token. Figure 4 offers a scheme with legends of the main components.

Fig. 3: The diffractometer-token used at the IJL

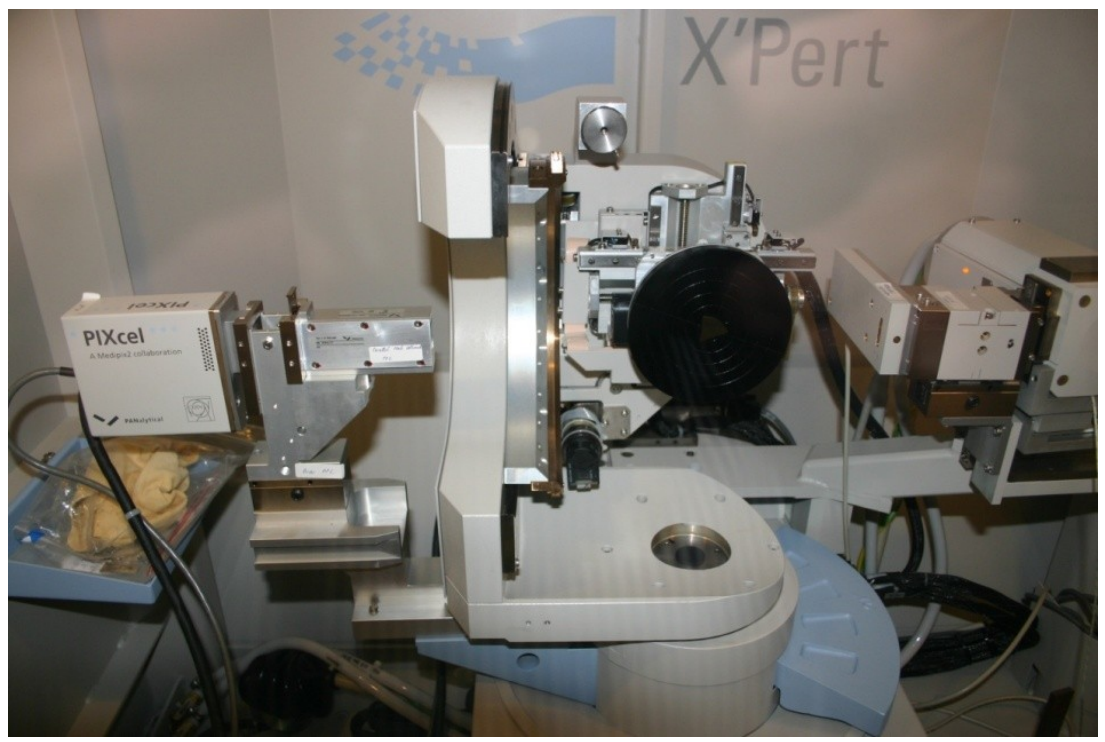

Fig. 4: Scheme of the main components of an X-ray diffractometer 


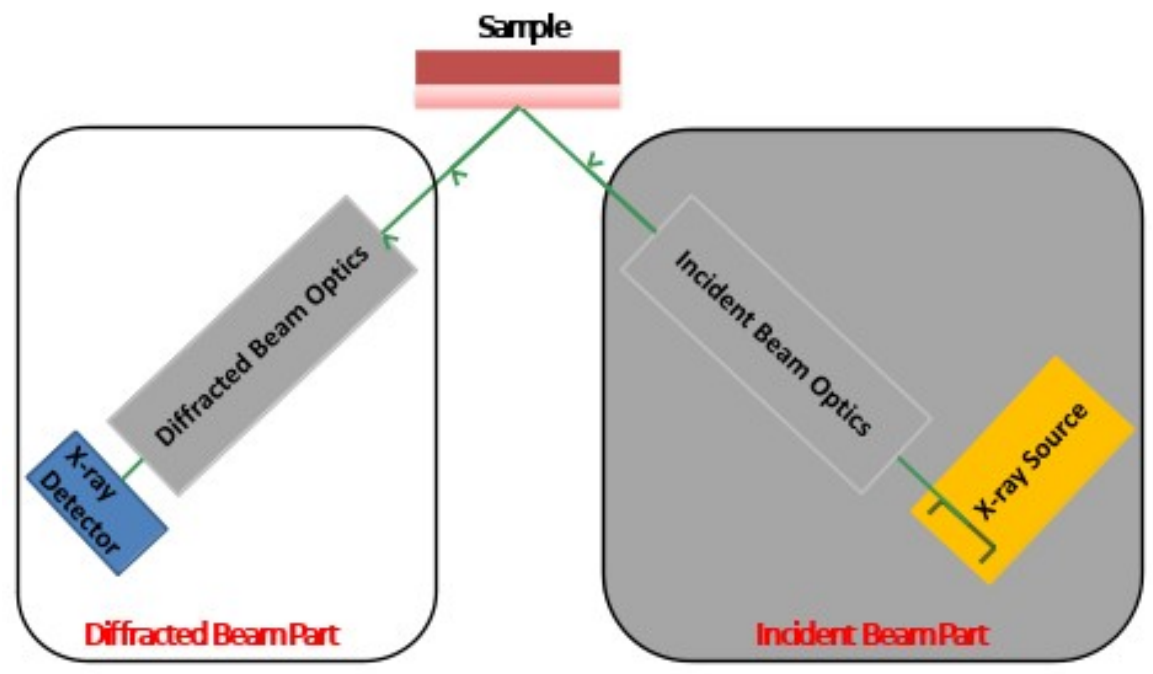

The components of this diffractometer-model are (from the right to the left):

(i) The $\mathbf{X}$-ray source. Here it is a source-token of the type 'Cu-anode sealed-tube'. The source produces an incident monochromatic X-ray beam with an intensity $\mathrm{I}_{\mathrm{inc}}$, which is directed on the crystalline thin-film sample to be analyzed.

(ii) The sample holder where the crystalline thin-film sample is laid down.

(iii) The X-ray detector. Here it is a detector-token of the type 'PIXcel detector', which is a second generation solid-state detector based on pixel technology. The detector collects the X-ray beam diffracted by the crystalline sample in a given direction, and indicates, for each angle, the diffracted intensity $\mathrm{I}_{\text {diff, }}$ that is, the number of diffracted X-ray photons.

Other instrumental modules are involved and play an important role.

(iv) A goniometer system. Its function is the determination of the relative positions of the source, the crystalline sample and the detector. The control of these positions is crucial for the interpretation of the instrumental outputs ${ }^{13}$.

(v) Some optical devices. They include: a monochromator, used to produce, as its name indicates, a monochromatic incident beam; and a collimator, used to filter the diffracted X-rays in order to collect only the $\mathrm{X}$-rays photons arriving at $2 \theta$ (see figure 2).

(vi) A diffractometer control software program. This program carries out all real-time instrument control functions. For example, it drives the goniometer motors, monitors the detector system, etc.

(2b) This model of diffractometer converts certain humanly performed operations (namely here: to put the crystalline thin-film on the sample holder; to switch the X-ray beam on; etc.), into one definite value of the mesurandum under interest (namely here: the value of the inter-reticular distance $d$ of a given thin-film). The conversion relies on some scientific theories, here the theory of diffraction of X-rays by crystals (which itself involves crystallographic theories and wave theories).

The passage from the above-mentioned humanly performed operations to a determined value $d_{1}$ of the mesurandum $d$, is realized through a scientific scenario, based on the Bragg's law, of the kind described above in point (1b), specified according to the contextual characteristics of the model: the monochromatic X-ray beam emitted by the $\mathrm{Cu}$-anode sealed-tube passes through the monochromator; the resulting monochromatic X-ray beam of wavelength $\lambda$ reaches the thin-film according to the direction $\theta$ prescribed by the goniometer; the proportional detector collects the scattered intensity for an interval of angles prescribed by the control software

\footnotetext{
${ }^{13}$ But cannot be even sketched here: The analysis of the corresponding calibration procedures would require another paper.
} 
program... And so is finally produced a graph as the instrumental output of the diffractometer, which is then interpreted in terms of a diffraction spectrum $\mathrm{I}_{\text {diff }}(\theta)$, from which the inter-reticular distance $d$ is finally inferred.

(2c) The instrumental outputs of a X-ray diffractometer are most of the time graphs. This is the case for the model used at the IJL. The corresponding graphs are interpreted as diffraction spectra $I_{\text {diff }}(\theta)$, that is, as the number of X-photons diffracted by the crystalline thin-film for different directions of the incident beam. Typically, the graphs present a peak of intensity for the Bragg's angle involved in the Bragg's law.

(2d) The scenario sketched above point (2b) - for which more or less detailed versions can be provided - is one part of the definition of the diffractometer-model. Its most detailed version provides the characterization of the diffractometer in optimal working (i.e., what is at best obtainable with a diffractometer-token generated according to this model). The other part of the definition of the diffractometer-model involves the possible deviations with respect to the optimal working that are anticipated according to the model. For example, a decrease over time of the intensity of the X-Ray beam delivered by the diffractometer is predicted.

In the present section, we have used the frame of Table 1, first constructed in relation to the case of the balance, to another instrumental device, namely, the X-ray diffractometer-token currently used at the IJL. We hope that the corresponding analyses have shown how Table 1 enables to achieve a fine-grained analysis of newlyconsidered instrumental devices, and have been sufficient to convince the reader of the interest of the conceptual tools we have introduced in this purpose.

Having characterized the measurer-token under interest - the X-ray diffractometer-token - we are now going to turn to the issue of its calibration. We start with the calibration of the diffractometer-token as a whole, using the simple exemplar (as summed up in Table 2) as a tool. At this level, our analysis will not go into the details, but we hope it will show the clarifying power of the simple exemplar as an analytic tool. Our aim is to give a sort of panoramic view of the situation concerning the calibration of the diffractometer-token as a whole (section 5.4), before to zoom in, and focus on one particular calibration step directed toward one particular component of the whole, the X-ray source-token (section 5.6).

\subsection{Calibration of the X-ray diffractometer as $a$ whole: an analysis by means of the simple exemplar used as a framework}

Let us now show how the simple exemplar of calibration, first constructed and illustrated relying on the simple case of the balance, can then be used as an helpful tool in order to understand more complex cases - here, the calibration of the X-ray diffractometer-token used at the IJL in the perspective of ends-measurements aiming at the determination of the inter-reticular distance $d$ of a crystalline thin-film. The simple exemplar is a useful tool, to the extent that it points to relatively well-predetermined places to look at, where something interesting should be found - or if not found, will nevertheless be instructive. To see how the simple exemplar of calibration plays this role, the reader has to go back to Table 2.

Table 2 first invites us to ask what the target of calibration is (first question of our four-questions frame). We have already answered to this question at the end of section 5.2, after having explained what has to be taken into account in order to provide an answer: the target is the X-ray diffractometer as a whole, because it is the X-ray diffractometer as a whole which is used in the end-experiments.

The second question of our four-questions frame calls attention to the presuppositions about the target of calibration, and Table 2 proposes two answers which obviously apply without any need of accommodation to the X-ray diffractometer: P1 and P2 hold. In other words: (i) the type and the model of the diffractometer are unproblematic (P1) - which, by the way, implies that the same holds for the type and the model of each component of the diffractometer; (ii) the diffractometer-token is assumed to be not deficient - which implies that each of its constituents works properly (P2).

Similarly, the answer, provided Table 2, to the question of the aim of calibration, applies as such to the calibration of the diffractometer as a whole: the aim of the calibration procedure of an X-ray diffractometer is to master the possible discrepancy between the diffractometer in optimal working and the diffractometer-token used in a given context in the perspective of certain end-measurements. The distinction between calibration tests and calibration operations also clearly applies here: to master the discrepancy means, first to evaluate, and second, if needed, to accommodate by means of some operations, the distance of the diffractometer-token from the optimal working as defined by the diffractometer-model. After examination of the operations currently performed at the IJL, it appears that both material and symbolic operations may be involved.

Table 2 furthermore invites to wonder if calibrations with etalons are undertaken or not in the practices under scrutiny, and the answer is positive: physicists of the IJL frequently use crystalline samples of known properties in order to evaluate the possible shift between their actual token of diffractometer in a given context and the 
optimal working as specified by the model. And of course, when such calibration tests with etalons are performed, presuppositions P3 (reliability) and P4 (adequacy) about the etalon are taken for granted.

Finally, Table 2 raises the question of blank calibration tests of an RX-diffractometer-token. The issue of blank calibration tests, applied to the diffractometer case, is perhaps not so straightforward at first glance. It will be discussed below, section 5.5.1, point (8).

All in all, relying on the simple exemplar of calibration as summed up in Table 2, it appears that most of the features previously well-characterized and developed in reference to the simple case of the balance, mutatis mutandis apply as well to the calibration of the newly-considered, more complex case of the X-ray diffractometer. To that extent we immediately achieve, thanks to the framework of the simple exemplar, a detailed characterization of central features of what is at stake in the calibration of a newly-encountered case.

There are, however, some specificities of the calibration of the X-ray diffractometer compared to the simple exemplar of calibration. Before to discuss some differences at the level of the details of the calibration procedure, let us consider, at the most general level, the main difference between the simple exemplar of calibration and the more complex case of the calibration of the X-ray diffractometer. The main difference comes from the clause that the diffractometer is a complex instrumental device. To understand this difference, we must specify the status of the complexity here involved. Such complexity must not be understood as an 'absolute' property of the X-ray diffractometer (the absolute property of 'being composed of parts'). It is a practical complexity, relative to the practitioners' concrete actions. Actually the balance itself could, like the diffractometer and like any other instrument, be decomposed as well in multiple parts - even if above, we have treated it most of the time as an 'atomic measurer' for the sake of simplification. If the diffractometer is treated here as a composed measurer rather than as an 'instrumental atom', it is because at the IJL, experimenters treat it as composed in their practices of calibration, in the sense that they perform different calibration tests directed toward different parts of the diffractometer. This 'spatial complexity' also implies a temporal complexity: the calibration of the diffractometer as a whole involves a long sequence of actions, which can be decomposed in multiple sub-sequences, or calibration steps ${ }^{14}$, more especially focused on this or that sub-part of the whole (with possible constraints on the order according to which the steps have to be implemented).

Such spatial and temporal complexity constitutes the main difference between the calibration of the X-ray diffractometer and the simple exemplar of calibration. A complete analysis of the calibration of a treated-ascomposed instrumental system, as is the X-ray diffractometer in current uses at the IJL, would require to scrutinize the multiple calibration steps involved, their order and possible inter-relations, and the way they are combined in global judgments of the type "the diffractometer as a whole is correctly calibrated (or not)". The construction of more complex exemplars of calibration would be needed to take these aspects into account. In what follows, we provide fragmentary insights on these aspects, through considerations about what we call "cocalibrations" (section 5.6). Note that regarding the aim of taking into account the complexity of calibration of a treated-as-composed measurer-token, the simple exemplar (Table 2), as well as the instrument-frame (Table 1), still remain helpful, to the extent that they can be used to analyze and illuminate each calibration step (see section 5.5 for an illustration applied to the case of the X-ray source).

Having stressed the complexity of the whole sequence of calibration of such a treated-as-composed measurer, we are now going to focus on one particular step of this complex sequence. This step, directed toward the X-ray source, shall be analyzed in details, using one more time the conceptual frames summed up in Table 1 and 2.

\subsection{One step in the calibration of the $X$-ray diffractometer as a whole: a calibration procedure directed toward the $\mathrm{X}$-ray source}

In the calibration step under scrutiny, the target of calibration is the X-ray source-token currently used at the IJL as a part of the diffractometer-token. Thus first of all, let use characterize this X-ray source by means of Table 1 .

\subsubsection{The X-ray source used at the IJL: an analysis by means of the instrument-frame}

The X-ray source, contrary to the instrumental devices considered so far, is not a measurer. To take this fact into account, Table 1 needs to be slightly adapted. Take the cell entitled: "Measuranda for the measurement of which the generic type is designed” (second column, line 2). Since the X-ray source is not a measurer, it is strictly

\footnotetext{
${ }^{14}$ This temporal complexity is almost always present in real procedures of calibration of a balance. In this respect as in many others, the characterization of the simple exemplar has to be understood as a simplification. This is obvious from the brief considerations, given above (section 4.6.3), about the procedures of 'full' calibration of a balance, which clearly show the temporal complexity involved, through the mention of multiple calibration steps (even if in this case, the different steps are directed toward different parameters rather than toward different parts of the balance).
} 
speaking not correct to say that it has been designed for the purpose of some determined measurements which typically intend to evaluate some specifiable measuranda. True, the X-ray source is designed to deliver an X-ray beam of a given intensity. Thus, the kind of quantity centrally and typically associated with an X-ray source is an $\mathrm{X}$-ray intensity. But this kind of quantity is not well described as a kind of quantity "typically involved in the measuranda”, as Table 1 mentions in its second column, line four. All in all, it would be more adequate to talk about the phenomena for the generation of which the X-ray source have been designed, and about the quantities typically involved in these phenomena. Hence we accommodate Table 1 accordingly: we replace "Measuranda for the measurement of which the generic type is designed" by "Phenomena for the production of which the generic type is designed", and "Kinds of quantities typically involved in the mesuranda" by "Kinds of quantities typically involved in the phenomena intended to be produced". Next we complete the accommodated frame for the X-ray source used at the IJL. Table 4 is obtained as a result. Table 4 gives a synthetic characterization of the target of the calibration step under scrutiny - the X-ray source - according to a frame which is by now, we assume, familiar enough to be provided without further comments, beyond the slight adaptations just described.

Table 4: A conceptualization of the RX-source-token used at the IJL, by means of a slightly modified version of the instrument-frame

\begin{tabular}{|c|c|c|}
\hline \multirow{4}{*}{$\begin{array}{l}\text { Generic } \\
\text { type }\end{array}$} & Generic name & Sealed-tube X-ray source \\
\hline & $\begin{array}{l}\text { Phenomena for the production of } \\
\text { which the generic type is designed }\end{array}$ & Monochromatic X-ray radiation \\
\hline & Fundamental scientific principle & $\begin{array}{l}\text { Generation of X-rays by irradiation of a material } \\
\text { target with a beam of high-energy charged particles } \\
\text { (such as electrons) }\end{array}$ \\
\hline & $\begin{array}{l}\text { Kinds of quantities typically involved } \\
\text { in the phenomena intended to be } \\
\text { produced }\end{array}$ & $\begin{array}{l}\text { X-ray intensity } \\
\text { X-ray wavelength }\end{array}$ \\
\hline \multirow[t]{4}{*}{ Model } & Name and description & Cu-anode sealed-tube \\
\hline & Scientific scenario & $\begin{array}{l}\text { A filament is heated to produce electrons which are } \\
\text { then accelerated in vacuum by a high electric field. } \\
\text { The high energy electrons are then directed toward a } \\
\text { metal target, namely a copper anode. The irradiation } \\
\text { of this Cu-anode by electrons generates a beam of X- } \\
\text { rays. }\end{array}$ \\
\hline & Instrumental outputs & - \\
\hline & $\begin{array}{l}\text { Deviations with respect to the optimal } \\
\text { working }\end{array}$ & $\begin{array}{l}\text { Decrease over time of the intensity of the X-Ray } \\
\text { beam generated by the source (ageing) }\end{array}$ \\
\hline
\end{tabular}

\subsubsection{A calibration directed toward the X-ray source: an analysis by means of the framework of the simple exemplar}

Table 5 below gives an overview of the results of our analysis concerning the calibration step directed toward the $\mathrm{X}$-ray source. The details of this analysis are provided below, in relation to the different numbers included in the cells of Table 5. In italicized text, we indicate the elements that are peculiar to this case, due to the two facts, first that the target of calibration corresponds to this particular model of X-ray source rather than another instrumental device (such as a balance), and second, that the calibration under scrutiny is performed in the perspective of these particular end-experiments rather than another ones. In underlined text, we stress the differences manifested by this calibration step with respect to the simple exemplar of calibration. Thus the underlined items direct the attention on aspects with respect to which the simple exemplar of calibration is not sufficient or needs adaptations.

Table 5: Analysis of one calibration step directed toward the X-Ray source-token used at the IJL by means of an elaborated version of Table 2 
Calibration in UNSI practices aiming at the determination of structural properties of mono-crystalline thin-films by means of an $R X$-diffractometer of the model described in Table 4 (1)

\begin{tabular}{|c|c|c|}
\hline $\begin{array}{l}\text { End- } \\
\text { measurements (2) }\end{array}$ & \multicolumn{2}{|c|}{$\begin{array}{l}\text { To assess the stability of a crystalline thin-film sample through time; to compare the structural quality } \\
\text { of one and the same sample at different moments }\left(t_{n} \text {, } t_{n+1} \text {, etc.), which implies to compare the height and }\right. \\
\text { width of the diffraction peaks obtained for this sample at } t_{n}, t_{n+1} \text {, etc. }\end{array}$} \\
\hline Target (3) & \multicolumn{2}{|c|}{ The $X$-ray source-token (see Table *4 for a details characterization) (3a); Not a measurer (3b) } \\
\hline $\begin{array}{l}\text { Presuppositions } \\
\text { (4) }\end{array}$ & $\begin{array}{l}\text { About the target (4a) } \\
\text { (P1) Unproblematic type } \\
\text { (P2) Non-defective token }\end{array}$ & $\begin{array}{l}\text { About the etalon } \\
\text { - }\end{array}$ \\
\hline Aim (5) & \multicolumn{2}{|c|}{$\begin{array}{l}\text { To master the obtained/optimal discrepancy of the intensity delivered by the X-ray source (5a) } \\
\text { Generalized definition: to master the 'obtained at } \mathrm{t}_{\mathrm{n}} \text { /obtained at } \mathrm{t}_{\mathrm{n}+1} \text { ' difference between the intensity } \\
\text { values, taking into account, as a reference point, the intensity value corresponding to the optimal } \\
\text { working (5b); if } \mathrm{t}_{\mathrm{n}} \text { corresponds to the optimal working, the general definition reduces to the } \\
\text { definition given for the simple exemplar, i.e., to (5a) }\end{array}$} \\
\hline \multirow[t]{4}{*}{ Procedure } & \multirow[b]{2}{*}{ 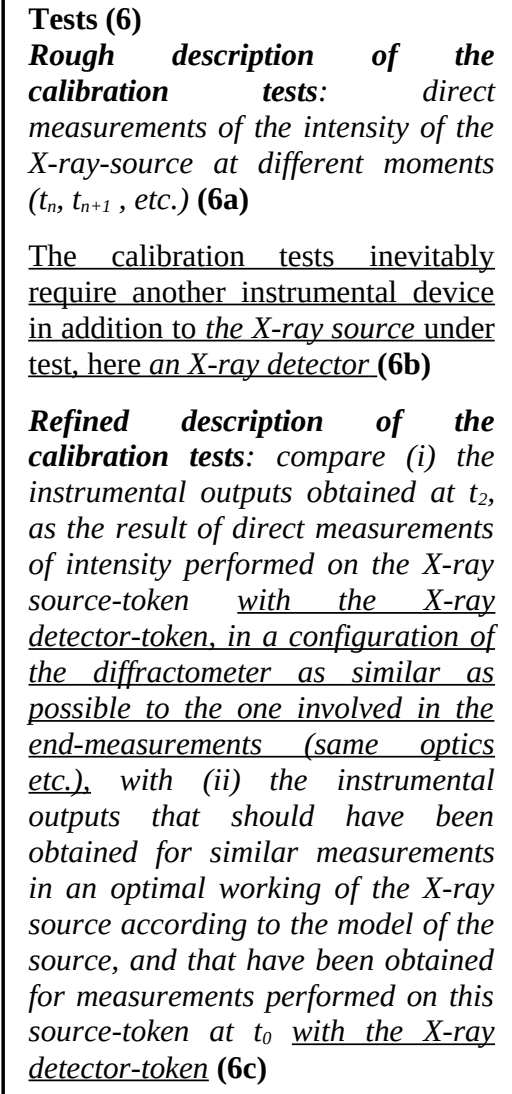 } & $\begin{array}{l}\text { Blank (8) } \\
\text { A blank calibration test of the diffractometer but not a } \\
\underline{\text { blank calibration test of the source }}\end{array}$ \\
\hline & & With etalon: - \\
\hline & \multirow[t]{2}{*}{ Operations (7) } & Material: - \\
\hline & & $\begin{array}{l}\text { Symbolic: mathematical correction: the diffracted } \\
\text { intensities of the diffraction spectra obtained in the end- } \\
\text { measurements are multiplied by a certain factor (7a) } \\
\text { The symbolic operations cannot be applied to the } X \text {-ray } \\
\text { source-token which is the target of the calibration. They } \\
\text { are inevitably applied to the instrumental outputs of the } X \text { - } \\
\text { ray-detector which plays the role of a measurer in the } \\
\text { calibration procedure directed toward the } X \text {-ray source } \\
\text { (7b) }\end{array}$ \\
\hline
\end{tabular}

(1) Our aim is to characterize a particular case of calibration in UNSI practices, so we must specify the first line of Table 2 accordingly (in italics, first line, second column). 
(2) As stressed section 4.3, the details of a particular activity of calibration directed toward one and the same instrument-token might differ according to the end-measurements. To that extent, the characterization of a particular activity of calibration must first of all examine the end-measurements.

The calibration step under scrutiny, that is, the calibration step of the diffractometer directed toward the X-ray source, is not systematically undertaken at the IJL. It is required only with respect to some types of endmeasurements (this gives an additional illustration of the crucial importance of the end-measurements, regarding not only the conception and relevant features of the calibration activity, but moreover its very existence). The calibration step under interest is required in relation to the second kind of end-measurements mentioned section 5.2. Such end-measurements intend to assess the stability of the thin-film sample through time. They therefore involve a comparison of the structural quality of one and the same mono-crystalline thin-film at different times (say $t_{1}$ and $t_{2}$ ). When such a comparison is projected, practitioners must care about the values of the diffracted intensities, and not just about the fact that there is an intensity peak at a certain angle, no matter its value (as it was the case for the determination of the inter-reticular distance at a given moment). Since the diffracted intensities depend on the incident intensity delivered by the X-ray source, a calibration step is required in order to control the intensity of the beam delivered by the X-ray source.

More precisely, to know whether or not the quality of a thin-film has been altered or not since its first production, practitioners must reiterate, at a subsequent time $t_{2}$, a structural characterization of the same kind as the one previously performed on the same thin-film at an anterior time $t_{1}$. The instrumental outputs of the experiments which provides the basis of such structural characterization are, as we saw, graphs interpretable as diffraction spectra $I_{\text {diff }}(\theta)$. So once the end-measurements have been performed at $t_{2}$, practitioners are left with (at least) two diffraction spectra, $\mathrm{I}_{\text {diff }}(\theta) \mathrm{t}_{1}$ and $\mathrm{I}_{\text {diff }}(\theta) \mathrm{t}_{2}$, that they have to compare. However, the height and width of the diffraction peaks recorded at $t_{1}$ and $t_{2}$ - from which the diffracted intensities at $t_{1}$ and $t_{2}$ must be derived - are directly comparable (say by superposition of the two graphs obtained at $t_{1}$ and $t_{2}$ for the same sample) only under the condition that, at $\mathrm{t}_{1}$ and $\mathrm{t}_{2}$, the $\mathrm{X}$-ray source-token delivered an incident beam having the same intensity $\mathrm{I}_{\text {inc }}$.

(3) This condition does not necessarily hold. Some accident or other unexpected event may have altered the production of X-ray photons by the source. More fundamentally, as indicated in Table 4 (last line), according to the specifications of the model of the $X$-ray source, a deviation with respect to the optimal working of the $X$-ray source is expected, corresponding to a decrease over time of the intensity of X-ray beam generated by the source ("ageing"). Consequently, with respect to any end-measurements for which the values of the intensities (or height and widths of the diffraction peaks) matter, a calibration procedure must be undertaken at a point or another, in order to control the intensity of the incident X-ray beam. The target of this calibration step is the Xray source-token (3a).

(4) The calibration step in question is undertaken under two presuppositions about the target of calibration (4a): the X-ray source-token is assumed to be (i) of unproblematic type (P1) and (ii) not defective (P2) (we already saw section 5.4 that it was the case for all the components of the X-ray diffractometer-token).

(5) The aim of this calibration step is to master (to evaluate in the calibration tests, and to take into account through the calibration operations) the difference between the values of the intensity delivered by the X-ray source, on the one hand at $t_{1}$, and an the other hand at $t_{2}$ (5a). Such an aim can be abbreviated as "to master the obtained/optimal discrepancy of the intensity of the X-ray source-token”, with some qualifications that will be provided below (see point (6)), once the calibration tests will have been specified.

(6) The calibrations tests undertaken in order to achieve the previous aim consist in direct measurements of the intensity delivered by the X-ray source at different relevant moments (the measurements are 'direct' in the sense that no diffracting sample is involved in the test) (6a).

Back to the aim of calibration (5). Having specified the calibration tests, let us come back to the aim of the calibration step directed toward the X-ray source. Two cases can be differentiated.

a/ Suppose that $t_{1}$ corresponds to the first setting up in the laboratory of a new X-ray source-token just after its manufacturing, and that the source-token presents no default with respect to the specifications of the model (practitioners of course check this conformity when they receive a new instrument). In that case, the $\mathrm{X}$-ray source-token at $\mathrm{t}_{1}$ coincides with the $\mathrm{X}$-ray source-model in optimal working. The direct measurements of intensity realized at $t_{1}$ indicate a maximal incident intensity value $\mathrm{I}_{\text {diff }}$ obtainable with this source-token. The diffraction spectra recorded at $t_{1}$ (typically with etalons) constitute a reference point which characterizes the optimal working regime. Referred to this time $\mathrm{t}_{1}$ at which the X-ray source-token coincided with the optimal working as defined by the model, the aim of the calibration tests and operations directed toward the same X-raysource-token at a subsequent time $t_{2}$ can be characterized, in complete conformity with the definition proposed above for the simple exemplar, as (5a): to master the obtained / optimal discrepancy - here the discrepancy of the intensity of a sub-part of the diffratometer as a whole, the X-ray source. If the X-ray source-token is, at $t_{2}$, 
still conform to the X-ray source-model in optimal working, the diffraction spectra obtained at $\mathrm{t}_{2}$ are directly comparable to the one obtained at $\mathrm{t}_{1}$ in optimal working (by simple superposition of the graphs).

b/ However, in practices at the IJL, the comparison is not always directly and explicitly referred to the 'initial' time where the source-token coincided with the optimal working. The comparison often contrasts what holds at a time $t_{3}$ with an anterior time $t_{2}$ where the source had already shifted from the optimal working. In such cases, our initial definition of the aim of calibration as the obtained / optimal discrepancy (here the decrease of the X-ray source-token intensity) must be slightly modified, in the sense of a generalization and an increased complexity. In such cases, the gap that practitioners aim to assess in the calibration test is, more generally (5b), an 'obtained at $t_{n} /$ obtained at $t_{n+1}$ ' difference. When $t_{n}$ corresponds to the optimal working, the latter general definition of the aim of calibration reduces to the definition (5a) given for the simple exemplar.

Actually, this general definition is still a simplification, since in fact, what is at stake is not just two moments $\mathrm{t}_{1}$ and $t_{2}$, but a whole temporal trajectory, which might involve an indefinite number of relevant moments, say $t_{0}$ (=optimal working), $t_{1}, t_{2}, \ldots, t_{n}$. For the sake of simplicity, however, in what follows, we shall reduce the problem of the calibration directed toward the X-ray source to a situation in which (i) only two moments are compared (say $\mathrm{t}_{2}$ and $\mathrm{t}_{0}$ ) and (ii) the first moment $\mathrm{t}_{0}$ corresponds to the optimal working. In such a situation, the aim of calibration coincides with the aim defined for the simple exemplar (i.e., to master the possible obtained / optimal discrepancy).

Back to calibration tests (6). As already stressed section 5.5.1, the target of calibration, the X-ray source, is not a measurer (3b): the X-ray source does not deliver instrumental outputs directly accessible as measurement results. The fact that the $\mathrm{X}$-ray source is not a measurer has repercussions at the level of the calibration tests and introduces, at this level, an important difference with respect to the simple exemplar (6b): the calibration tests of the X-ray source inevitably require, in addition to the X-ray source under test, another instrumental device which plays the role of a measurer - that is, which records the intensity delivered by the X-ray source-token at a time $t_{2}$ close to the end-measurements. In other words, the calibration tests inevitably require an $X$-ray detector. In the calibration tests performed at the IJL, this additional device is the X-Ray detector-token which is part of the diffractometer and is intended to be used in the end-measurements.

Taking that into account, a refined description of the calibration tests goes as follows (6c). Direct measurements of intensities are performed, at $\mathrm{t}_{2}$, on the X-ray source-token with the X-ray detector-token, in a configuration of the X-ray diffractometer as similar as possible to the one involved in the end-measurements (e.g., using the same detector, the same optics, etc.). The instrumental outputs of the detector obtained at $\mathrm{t}_{2}$ (which coincide with the instrumental outputs of the diffractometer obtained at $\mathrm{t}_{2}$ ) are then compared to the ones that should have been obtained for an optimal working of the X-ray source according to the model of the source, and that have actually been obtained at $\mathrm{t}_{0}$ with this $\mathrm{X}$-ray source-token, this detector-token, these token of optics, etc.

(7) Having described calibration tests, let us turn to the issue of calibration operations.

If no significant difference is found between the intensity values obtained at $t_{0}$ and at $t_{2}$, no calibration operation is required. The diffraction spectra obtained in the past at $\mathrm{t}_{0}$ are directly comparable to the diffraction spectra that will be obtained in subsequent end-measurements at a time close to $t_{2}$. A simple superposition of the spectra at $t_{0}$ and $t_{2}$ enables to compare the values of the diffracted intensities and, on this basis, to decide whether or not the quality of the mono-crystalline thin-film has been altered between $t_{0}$ and $t_{2}$.

If, to the contrary, a significant difference appears between the X-ray intensities at $t_{0}$ and $t_{2}$, then, some calibration operations must be applied. In the present case, they correspond to symbolic operations (7a) applied to the instrumental outputs of the X-ray diffractometer (or, equivalently, to the instrumental outputs of the X-ray detector). More precisely, the diffracted intensities of the diffraction spectra obtained in the end-measurements are multiplied by a certain factor (a proportionality rule is applied). The fact that the X-ray source which is the target of calibration is not a measurer introduces a difference, with respect to the simple exemplar, concerning the way the symbolic operations are applied (7b). The proportionality factor cannot be applied directly to the instrumental outputs of the X-ray source which is the target of the calibration test, since these outputs are not directly accessible but must be recorded by the mediation of the X-ray detector. The mathematical correction that is needed, in order to take into account the intensity decrease of the X-ray source-token, are thus inevitably applied, not directly to the target of calibration, but to the instrumental outputs of another instrumental device which is a measurer, here the $X$-ray detector.

Can we say that once the symbolic operations have been applied, practitioners have calibrated the $X$-ray source? A hesitation might occur, which did not occur in the case of the simple exemplar of calibration. This is because the source is not a measurer and the operations are symbolic operations. Consequently, these operations are inevitably applied to the instrumental outputs of the detector rather than directly to the source. Since the 
corrections are applied to the outputs of the detector, we might hesitate, as far as calibrating operations are concerned, to say that practitioners have calibrated the source. However, nothing forbids to say that practitioners have calibrated the source by applying the symbolic corrections to the outputs of the detector (or equivalently, by applying intellectual corrections to the outputs of the diffractometer). Actually, this seems a perfectly adequate way of conceptualizing the situation. To be convinced, imagine a structurally similar situation which differs only in that material operations are involved rather than symbolic operations. We would have no problem to say that practitioners have calibrated the $X$-ray source (since the material operations would be physically applied directly to the source). The fact that there is no fundamental structural difference between these two cases encourages to conclude that it is not only permitted, but also appropriated, to say that through the intellectual corrections applied, practitioners have calibrated the $X$-ray source (or more exactly, the intensity of this source).

(8) Finally, how should the calibration tests here involved be situated with respect to the two species previously distinguished, namely blank calibration tests and calibration tests with etalons? Clearly, no etalon is involved in the calibration step under discussion, so the first species is not relevant. But what about the situation of the calibration step here involved with respect to a calibration test of the blank type? The answer is not straightforward and implies partly conventional terminological/conceptual decisions that can be motivated but are not universally compelling. We experienced divergences at this level in the PratiScienS team during the course of our inquiry. Let us formulate and motivate the decisions about which we finally agree.

First, the calibration procedure above-described can be identified with a blank calibration, to the extent that it conforms to the main elements involved in the definition, adopted above section 4.4.1, of what a blank calibration is. The calibration tests under scrutiny are indeed (i) measurements of the same kind as the endmeasurements: same X-ray source-token, same X-ray detector-token, same optics (in a word, same diffractometer-token); same kind of measured-quantity (X-ray intensity); and (ii) measurements undertaken in the absence of any object of the same kind as the object the end-measurements aim to characterize (a diffracting crystalline sample). The end-measurements and the calibration tests are supposed to differ only in regard to the condition 'with/without a diffracting sample', all other things being equal. On this basis, it seems adequate to describe what is at stake as a blank calibration of the diffractometer.

Can we also talk about a blank calibration of the $X$-ray source? It does not seem meaningful, despite the fact that the calibration step is indeed directed toward the $X$-ray source. This is because a blank calibration test of an instrument consists in recording the instrumental outputs delivered by this instrument in the absence of a measured-object of the kind involved in the end-measurements. Yet an X-ray source does not deliver any directly recordable instrumental outputs. No instrumental output can be recorded with an X-ray source alone. As already stressed above point (6), another instrumental device, here a measurer able to record an X-ray intensity, is required. We conclude that it is not appropriated to talk about a blank calibration of the $X$-ray source.

Yet, to describe the calibration tests under discussion as blank calibration tests of the X-ray diffractometer seems to miss an important aspect of these tests, namely that they intend to check properties of one particular component of the diffractometer, the $X$-ray source. If we want to put emphasis on this aspect, we can, leaving aside the adjective "blank", use - as we did so far - the alternative expression "calibration tests directed toward the X-ray source". We prefer to avoid any formulation in terms of a calibration test of the X-ray source, because although the intention is indeed to test the $X$-ray source, in concreto, what is tested is never the source alone, but always the source plus the detector (we will come back to this point in the next section). This is why, so far, we have used the perhaps at-first-sight pointlessly complicated expression "calibration test directed toward the Xray source" rather than "calibration test of the X-ray source".

To recap, the calibration tests under scrutiny can be characterized, either as calibration tests (neither blank, nor with etalons) directed toward the $\mathrm{X}$-ray source, or as blank calibration tests of the $\mathrm{X}$-ray diffractometer. Generalizing beyond the particular case of the X-ray source, it is meaningless to talk about a blank calibration of a non-measuring device.

\subsection{Some general epistemological implications for the calibration of a treated-as- composed instrumental system}

Let us draw some general lessons from the particular case of calibration in X-ray experiments at the IJL.

There are calibration tests which differ from the simple exemplar in that their target $T$ is not a measurer. This has been illustrated above by the case of the X-Ray source, but the point can be generalized. For example, it applies as well to the optical devices involved in the diffractometer. In such cases, there is a dissociation between the targeted object $\mathrm{T}$ of the calibration test on the one hand (in our example: the X-ray source-token), and on the other hand, the device which provides the values of the mesuranda (in our example: the X-ray detector-token which provides the values of the intensity delivered by the source). 
This dissociation at the level of the calibration tests has repercussions at the level of the calibrating operations when the latter are symbolic operations. Indeed, the corresponding corrections (in our example: the application of a proportionality factor) cannot be applied directly to the instrumental outputs of the device which is the target of the calibration test (in our example: the X-ray source), since these outputs are not directly accessible but must be recorded by the another device which plays the role of the measurer (in our example: the X-ray detector). The symbolic operations introduced in order to take into account the obtained / optimal discrepancy related to the non measuring device under test (the decrease of the X-ray source intensity) are thus inevitably applied to the instrumental outputs of another device than the target $\mathrm{T}$ of calibration, that is, to a measurer (the X-ray detector)

This situation is not deprived of epistemological consequences. Indeed, the individual X-ray detector could, of course, itself have shifted from the optimal working corresponding to its model. But if the detector-token involved in the calibration test of the source-token is not properly calibrated, then, the calibration test of the optimal / obtained discrepancy of the intensity values of the X-ray source is not be reliable. With such considerations, we begin to meet the holistic features of the calibration procedure of a complex (i.e., treated as composed) instrumental device. What is actually tested, in the calibration test of a non measuring device, is not just this non measuring device-token at $t_{2}$, but the set 'non-measuring device-token at $t_{2}+$ measurer-token at $t_{2}$ '. Thus in situations of this kind, we must distinguish the intended target and the effective target of the calibration test. The intended target $\mathrm{T}$ of calibration (in our example: the X-ray source-token at $\mathrm{t}_{2}$ ) does not coincide with the effective target (in our example: the X-ray source-token at $t_{2}$ plus the $X$-ray detector-token at $t_{2}$ ). The calibration test of the effective target reduces to a test of the intended target only if the detector is indeed itself wellcalibrated. So a prior calibration test of the detector-token seems to be required.

However, as a matter of fact, at the IJL, practitioners do not perform themselves the calibration tests and operations directed toward the detector-token. This part of the diffractometer is, according to its model, much less subject than the source to an important drift over time. So in routine practices, IJL experimenters do not perform any calibration tests that take the detector as their target. But beyond the particular case of experimental practices at the IJL, the situation just described points to a widespread interesting configuration with respect to calibration practices: something like a co-calibration of some instrumental modules involved in a treated-ascomposed instrumental system. Let us elaborate this point a little bit further.

Consider a complex instrumental system (say $C$ as complex), treated-as-composed of multiple sub-modules, among which one finds at least one instrumental device which is not a measurer (say $D$ as Device) and one instrumental device which is a measurer (say $M$ as measurer). Suppose moreover that the instrumental outputs of $C$ and the instrumental outputs of $\mathrm{M}$ coincide. Any calibration test of $D$ inevitably requires, in addition to the non-measuring device under test, an $M$. This introduces a difference between the intended target of calibration $(D)$ and the effective target of calibration $(D+M)$. Consequently, all the conclusions about the calibration of $D$ hang on the issue of whether or not $M$ is itself properly calibrated (and a fortiori not defective). How can practitioners deal with such situations?

A common practice is to perform calibration tests of $C$ with some etalon. Imagine that the instrumental outputs of $C$ obtained in such calibration tests are interpretable in a way which coincide with the expected characteristics of the etalon. If this is the case, all the sub-modules involved in C (notably $D$ and $M$ ) will be considered as correctly calibrated. In such a situation, we can consider that the measurer $M$ serves as a means to test the adequate calibration of the non-measuring device $D$, and reciprocally, that $D$ serves as a means to test the adequate calibration of $M$. Such a scheme can be characterized as a co-calibration.

Alternatively, imagine that the instrumental outputs obtained with $C$ in the previous calibration tests are not the expected ones. In such a situation, it is often required to find the source of the problem. In the example of the Xray diffractometer, an in principle possibility (although not always available in practice, and not available at the IJL) would be to use several (say three) X-ray detectors and to test the intensity of the X-ray source with each of them at three moments close to one another. If the instrumental outputs of two of them coincide and the outputs of the third are very different, practitioners will suspect that it is the third detector, and not the source, which is not correctly calibrated. If the instrumental outputs of the three detectors coincide and correspond to a value of the intensity which inferior to the value in optimal working, practitioners will suspect that the source has drifted from its optimal working. And so on. In such a situation, we can talk of a co-calibration of the source and the three detectors.

This leads us back to the holistic features of the calibration procedure directed toward a treated-as-composed instrumental system. When dealing with a complex instrument $C$, practitioners have to perform a multiplicity and a long sequence of calibration tests, successively directed, from an intentional point of view, toward this or that sub-part $S, S$, etc. of the instrument. But some of the testing steps actually performed inevitably involve other elements than just the intentional target of the calibration test, and moreover, the various calibration steps must be performed one after the other. At each step of the whole calibration sequence focused on a particular 
instrumental module $S$ taken as the intentional target $\mathrm{T}$ of the calibration test, practitioners think as if all the other modules $S$ ', $S^{\prime \prime}$, etc. coincided with the optimal working (or more generally with some other determined reference). And so on from step to step, when permuting the instrumental module under test and the other assumed-as-optimal modules. A ceteris paribus condition must be presupposed all along the temporal process of the calibrating sequence: practitioners must assume that the obtained / optimal discrepancy related to $S$ does not vary during the evaluation of the obtained / optimal discrepancy related to $S^{\prime}$, and so on. Of course, this assumption is not a specificity of calibration procedures with respect to other scientific procedures. In particular, scientists must assume that the ceteris paribus condition holds, not just throughout the calibration sequence, but also subsequently, during the temporal interval which separates the end of the calibration sequence and the end of the end-measurements. But although the ceteris paribus assumption is not specific to calibration procedures, it has to be stressed that the more a calibration sequence is long and composed of a high number of delicate steps, the more the ceteris paribus clause can fail to apply in fact.

\section{Conclusions}

\subsection{Concrete insights on calibration gained so far}

In this paper, we have restricted our attention to the situation which seemed at first sight the less problematic with respect to calibration, namely, calibration in UNSI practices. In other words, calibration has been analyzed from the standpoint of scientific practitioners who are users of already well-designed and well-mastered instruments and explore relatively well-known phenomena by means of such instruments.

In order to clarify the nature of this kind of practice and to grasp its internal logic, we have constructed, as a first step, a simple exemplar of calibration in UNSI practices, illustrated by the case of the equal-arm balance. The simple exemplar in UNSI practices is defined by the following features. The target $\mathrm{T}$ of calibration is a measurer-token. The calibration of the measurer-token involves two presuppositions about the measurer-token: (P1) the measurer-model (and thus a fortiori the measurer-type) is not problematic; and (P2) the measurer-token is not defective (no breakdown). The aim of a calibration procedure directed toward a measurer-token $\mathrm{T}$ under presuppositions $\mathrm{P} 1$ and $\mathrm{P} 2$ is to master the gap between the values obtained with the measurer-token and the values that should have been obtained in the optimal working defined by the specifications of the measurermodel. The procedure through which the aim is achieved typically involves two steps: first calibration tests, which can be blank tests and/or tests with etalons; second, if needed - depending on the result of the tests calibration operations, which can be material and/or symbolic operations. In calibration tests with etalons, two presuppositions are endorsed about the etalon: the etalon is (P3) reliable and (P4) adequate, that is, sufficiently similar to the object under study in the end-measurements.

Next, we turned to a more complex case of calibration in UNSI practices, the calibration of an X-ray diffractometer in a nanoscience laboratory. Our intention, with the study of this more complex case, was twofold: (i) to show the working power of our conceptual framework for the analysis of different, more complex cases; and (ii) to introduce more complexity into the picture (in agreement to our strategy to go from the simple to the complex), by characterizing some features of more complex calibration procedures in UNSI practices that are not captured by the simple exemplar. Two such features have been put forward, and some of their epistemological implications have been analyzed. The first feature is: the target $\mathrm{T}$ of calibration can be a treatedas-composed instrumental system (this is certainly the most common case in scientific practices of calibration). As a consequence, the calibration of $\mathrm{T}$ is a (more or less) long sequence of multiple calibration steps. This raises the question of the relations between the multiple calibration steps and the calibration of the measuring system as $a$ whole. The second feature is: the target $\mathrm{T}$ of a calibration activity can be a non-measuring instrumental token. As a consequence, another instrumental token of the type "measurer" is inevitably involved in the calibration tests of T. Taking into account and combining the implications of these two features, we have been led to direct the attention toward the holistic character of the calibration of a treated-as-composed measurer as a whole, to introduce the idea of co-calibrations, and to consider some epistemologically important aspects of cocalibrations, namely, the non coincidence of the intended and effective target of calibration, and the ceteris paribus condition that must be assumed all along a sequence of co-calibration.

The two last paragraphs sum up the main substantial results about calibration achieved in the present article. We see these substantial results as concrete insights gained so far about calibration, thanks to the conceptual framework we have elaborated. This conceptual framework itself is, of course, another result of the article, of a more 'formal' or 'structural' kind. As a conclusion, and as promised in section 1.3, it is now time to come back to this framework and to revisit its main features.

\subsection{Revisiting the conceptual framework}


Now that the framework has been put to work, we are in a position to specify further its nature (what do we mean by a conceptual framework?), its status (what provides to a given characterization the status of a framework?), its scope (what is the domain of application of the different pieces of the framework?), and its value (what kind of work is accomplished by the framework?).

By a conceptual framework, we mean a set of more or less closely interrelated categories and questions, intended to work as conceptual tools and as guides for the analysis of a certain targeted subject matter (here, calibration). This implies that the corresponding categories help to understand and to classify multiple particular cases at first glance susceptible to be instances of the targeted subject matter. These categories must be both sufficiently general to apply to a multiplicity of particular cases (possibly with some adaptations), and sufficiently welldefined to drive efficiently and illuminate the analysis of newly-encountered cases. The latter specifications provide elements of answer to the two questions of the framework's nature and expected value.

The categories of the four-question frame (target, presuppositions, aim and procedure of calibration), the bold part of Table 1 , and Table $2^{15}$, can be considered as three conceptual frameworks in the previous sense - or, if one prefers, as three components of the same framework. The three components are of course not independent. The third one (the simple exemplar of calibration) relies on both the first one (since it uses the categories of the target, presuppositions, aim and procedure) and on the second one (since it uses the categories of the instrumentframe, such as the notion of measurer and the type/token distinction).

Further reflections about the relations between the three components enable to specify what provides to a given characterization the status of a framework, and by doing so, to offer a more complete explication of what is meant by a framework, as well as a more precise explanation of the kind of work the proposed framework is expected to accomplish. What has been called all along of this article "the framework of the simple exemplar of calibration" (summed up in Table 2) uses what has been called the "four-question frame", and gives determinate answers to each question of the target, the presuppositions, the aim and the procedure. To the extent that the simple exemplar of calibration gives determinate answers to the four questions, we could be tempted to consider that the simple exemplar of calibration is not rightly characterized as a framework (in the common sense of a form, that is, of a deprived-of-content shape or structure intended to be applied to some content related to calibration), but should better be characterized as a substantial description of calibration. So is the simple exemplar of calibration a framework in the sense of a form (intended to be applied to some substance), or is the simple exemplar a content (a substantial characterization of calibration)? Our answer is: the frontier between a framework understood as a form, and a substantial characterization understood as a content, is relative and context-dependent; in particular, it depends on the purpose of the analyst.

Let us illustrate. Table 2 indeed provides a substantial (admittedly not exhaustive) characterization of calibration in UNSI practices relatively to some instances (the simple instances for which it appears that Table 2 directly applies without any need of accommodation). Relatively to these instances, the simple exemplar 'tells us something' about calibration; it is not just a means in order to access to some knowledge about calibration. But relatively to other, more complex instances of calibration in UNSI practices - such as some calibration procedures in X-ray experiments conducted at the IJL - Table 2 plays the role of a framework in the sense of a form, and works as a guiding and enlightening benchmark, as we hope to have convinced the reader in part 5. The value of Table 2 lies in these two kinds of contributions taken altogether.

Let us be still more precise and, by the way, give the recipe of the use of the framework as it stands. Faced with a new instance of activity that could be identified with a calibration (for example: X-rays experiments described in part 5), use Table 1 and Table 2 as frames, and examine to what extent they directly apply or must be accommodated.

As far as Table 2 applies to the newly-scrutinized case, Table 2 provides both a guiding frame and a substantial characterization. More exactly, it provides (i) a guiding frame (for the X-ray example, see Table 5, items in black), which easily leads to a clear understanding of what is at stake - in the X-ray example, an understanding of most aspects of the calibration of the X-ray diffractometer as a whole (see section 5.3), and an understanding of some aspects of the calibration of the X-ray source (see Table 5, items in italics). To the extent that Table 2 used as a frame drives this understanding, Table 2 offers - or at least works as a guide for the easy acquisition of - (ii) a substantial characterization of the newly-encountered case.

As far as Table 2 does not apply as such to the newly-scrutinized case, Table 2 nevertheless works as a benchmark, in reference and by contrast to which significant differences can be circumscribed and characterized (see Table 5, highlighted in underlined items).

\footnotetext{
${ }^{15}$ We could add to this list the mapping of scientific practices sketched in section 3.1.3. This mapping is indeed an integral important part of what we identify with our framework. However, in this paper, only very sketchy elements have been provided.
} 


\subsection{Scope of the framework}

It remains to discuss the scope of our framework, that is, the domain in which the framework is expected to do some valuable job. Actually, the scope differs according to the components of the framework.

(i) Scope of the four-questions frame. The intended scope, and the fecundity, of the four-questions frame go much beyond the case of calibration in UNSI practices. The broad applicability is straightforward: obviously, the four questions can be applied to any activity candidate to calibration. The fecundity can only be claimed, but not showed, since in the present paper, only calibrations in UNSI practices have been discussed.

(ii) Scope of the instrument-frame. The bold part of Table 1 is applicable as such to any socially-stabilized measurer and is a clarifying tool for a fine-grained grasp of any measurer in UNSI practices (as we attempted to show that it was the case for some of them, in sections 3.3 .6 and 5.4). In relation to some measurers, complements may be relevant. When the aim is to study the calibration of a treated-as-composed instrument, for example, it may be interesting to add some cells to Table 1, dedicated to the specification of the instrumental sub-modules which play a role in the calibration activity. In addition to be a fruitful tool for the analysis of already-standardized measurers, Table 1 is not deprived of interest when we turn to standardized non-measuring devices. In the latter case, Table 1 serves as a benchmark but needs some accommodations - of the kind introduced in Table 4, for the particular case of the X-ray source.

(iii) Scope of the framework of the simple exemplar. Table 2 has the status of an exemplar, which means (as indicated in section 3.2.2) that Table 2 is not intended as an exhaustive characterization of any domain of calibration activities, nor as a set of necessary and sufficient conditions to categorize an activity as a calibration. Table 2 applies to calibration in UNSI practices, in the sense that Table 2 is relevant and fruitful as a tool for the understanding of calibration in UNSI practices, even if all elements of Table 2 do not directly apply as such to the calibration instance under study. We hope to have shown, through our analyses of the calibration of a balance and of an X-ray diffractometer, that at least in the domain of UNSI practices, the simple exemplar is a powerful analytic tool, which efficiently guides the achievement of a substantial characterization of the particular cases under scrutiny. We can add that the simple exemplar summed up in Table 2 is also very useful for the analysis of calibration in other kinds of scientific practices - although to argue this point is not possible in the volume of this article.

\subsection{Relation of the framework to science in practice}

Finally, some readers might perhaps wonder "how generating the conceptual framework relied upon the details of experimental practice" and "how the resulting conceptual framework differs from the product of armchair conceptual analysis" 16 . These are not easy questions. We are convinced that the framework could not have been provided "by merely thinking about calibration", but it is not so straightforward to argue. True, we can repeat that we started with examples issued from contemporary scientific activities as practiced by our physicist colleague C. Dufour and as observed by our ethnographer colleague C. Allamel-Raffin (see section 1.3). We can add that faced with difficulties, we again and again go deeper in the details and asked questions to the relevant scientists involved in the activities of calibration under scrutiny. But this is of course not enough to argue convincingly that the anchorage in 'science as it is performed in practice' made a significant difference, at the end of the day, regarding the conceptual framework we finally retained as the most adequate one. To give more convincing arguments, we would have to reconstitute at least bits of the genetic "long and sinuous process" process through which the final form of the framework have been elaborated (see section 1.4); we would have to focus more especially on hesitations, difficulties, blind alleys, and so on, and to explain how we finally succeeded to surmount them. However, such a task is not only impossible to realize in the available volume of this paper; it is moreover very difficult to achieve for fundamental reasons. This is because when a new version of framework is indeed better that the previous one, it leads to a restructuration of the understanding of the whole situation, from which results a neat relief at the moment, but which also implies that in the next step, the anterior difficulties are erased, and hence forgotten, so that from step to step, in the longer run, they are, to say the least, very hard to reconstruct. We are well-aware that the back and forth movement from practices to successive versions of the framework, and the corresponding important amount of work invested along the path, is difficult to perceive in the end-product. However, from now, what seems the most important is the value of the endproduct for further investigation of calibration by other researchers, and we are confident that the framework is valuable in this respect.

We have attempted, above, to provide explicit indications about the fruitfulness of our simple exemplar of calibration, and in particular, to provide explicit insights about the way the simple exemplar is revealing when applied to newly-encountered instances of calibration. However, no explicit formulation of this kind will ever be

\footnotetext{
${ }^{16}$ As Sally Riordan, as reviewer of a first version of this article, relevantly wondered.
} 
able to replace a personal experience 'in the first person'. The best way to be convinced of the fecundity of the framework is to put it at work in practice. We encourage readers interested in the topic of calibration to use our framework to analyze the cases in which they are interested. Try and see! More generally, we hope that the present work will inspire scholars to consider the significant but neglected topic of calibration.

\section{Acknowledgments}

We would like to express our gratitude to Karine Dumesnil, who, as a colleague of Cathy Dufour at the IJL, spent a lot of time to discuss calibration with us after Cathy passed away.

The present paper benefited from discussion with audiences at the Third Biennial Conference of the Society for Philosophy of Science in Practice (SPSP) in Exeter (June 2010), as well as at the International Conference “Dimensions of Measurement” organized by Alfred Nordmann and Oliver Schlaudt in Bielefeld (March 2013). The authors are moreover grateful to several anonymous reviewers, whose suggestions greatly contributed to improve the article.

Finally, we wish to thank the institutions that have supported or support the PratiScienS' project, namely: the ANR (Agence Nationale de la Recherche); the MSHL (Maison des Sciences de l'Homme Lorraine); the Région Lorraine, and the LHSP - Laboratoire d'Histoire des Sciences et de Philosophie - Archives Henri Poincaré (UMR 7117 of the CNRS).

\section{References}

Andersen, H. (2013; forthcoming). Epistemic dependence in contemporary science: practices and malpractice. In Soler, L. et al. (Eds.), Science After the Practice Turn in Philosophy, History, and the Social Studies of Science. London: Routledge.

Boumans, M. (2004). The Reliability of an Instrument. Social Epistemology, 18(2-3), 215-246.

Boumans, M. (2006). The Difference between Answering a 'Why' Question and Answering a 'How Much' Question. In Kueppers, J., Lehnard, J., \& Shinn T. (Eds.), Simulation: Pragmatic Construction of Reality (pp. 107-124). Dordrecht: Springer.

Boumans, M. (2007). Invariance and Calibration. In Boumans, M. (Ed.), Measurement in Economics (pp. 231248). London: Academic Press.

Bourget, M.-N., Licoppe, C., \& Sibum H. O. (2002). Instruments, Travel and Science: Itineraries of Precision from the Seventeenth to the Twentieth Century. London: Routledge.

Buccianti, R., Cibien, M., Mari, L., \& Rebaglia, B. I. (2009). Measurement and calibration: considerations based on the International Vocabulary of Metrology (VIM, $3^{\text {rd }}$ Ed.) and related standards, XIX IMEKO World Congress, Fundamental and Applied Metrology, September 6-11, 2009, Lisbon, Portugal.

Carnap, R. (1995 [1966]). An Introduction to the Philosophy of Science. New York: Dover Publications.

Chang, H. (2004). Inventing Temperature: Measurement and Scientific Progress. New York: Oxford University Press.

Chang, H. (2013; forthcoming). Epistemic Activities and Systems of Practice: Units of Analysis in Philosophy of Science after the Practice Turn. In Soler, L. et al. (Eds.), Science After the Practice Turn in Philosophy, History, and the Social Studies of Science. London: Routledge.

Chang, H. \& Yi, S. W. (2005). The Absolute and its Measurement: William Thomson on Temperature. Annals of Science, 62, 281-308.

Collins, H. M. (1992 [1985]). Changing Order: Replication and Induction in Scientific Practice. Chicago: University of Chicago Press.

Collins, H. M. (2004). Gravity’s shadow. Chicago: University of Chicago Press.

Franklin, A. (1986). The Neglect of Experiment. Cambridge: Cambridge University Press.

Franklin, A. (1990). Experiment: Right or Wrong. Cambridge: Cambridge University Press.

Franklin, A. (1997). Calibration. Perspectives on Science, 5(1), 31-80. 
Harris, T. (2003). Data Models and the Acquisition and Manipulation of Data. Philosophy of Science, 70(5), 1508-1517.

Heering, P. (2005). Weighing the Heat: The Replication of the Experiments with the Ice-Calorimeter of Lavoisier and Laplace. In Beretta, M. (Ed.), Lavoisier in Perspective (pp. 27-41). München: Deutsches Museum.

Licoppe, C. (1996). La formation de la pratique scientifique. Le discours de l'expérience en France et en Angleterre (1630-1820). Paris: La Découverte.

Livengood, J. (2009). Why was M. S. Tswett's chromatographic adsorption analysis rejected? Studies in History and Philosophy of Science , 40(1), 57-69.

Mallard, A. (1998). Compare, Standardize and Settle Agreement: On Some Usual Metrological Problems. Social Studies of Science, 28(4), 571-601.

Mari, L., \& Sartori S. (2007). A relational theory of measurement: Traceability as a solution to the nontransitivity of measurement results. Measurement, 40, 233-242.

O'Connell, J. (1993). Metrology: The Creation of Universality by the Circulation of Particulars. Social Studies of Science, 23(1), 129-173.

Ramsey, J. (2007). Calibrating and constructing models of protein folding. Synthese, 155, 307-320.

Rasmussen, N. (1993). Facts, artifacts, and mesosomes: Practicing epistemology with the electron microscope. Studies in History and Philosophy of Science (Part A), 24(2), 227-265.

Rossi, G., Crenna, F., \& Panero, M. (2005). Panel or jury testing methods in a metrological perspective. Metrologia, 42, 97-109.

Schickore, J. (2007). The Microscope and the Eye. Chicago: Chicago University Press.

Sibum, H. O. (1995). Reworking the Mechanical Value of Heat: Instruments of Precision and Gestures of Accuracy in Early Victorian England. Studies in History and Philosophy of Science, 26(1), 73-106.

Skipper, R. A. (2004). Calibration of Laboratory Models in Population Genetics. Perspectives on Science, 12(4), 369-393.

Tal, E. (2011). How Accurate is the Standard Second? Philosophy of Science, 78(5), 1082-1096.

VIM (2008), International vocabulary of metrology - Basic and general concepts and associated terms (VIM), Joint Committee for Guides in Metrology.

Werker, C., \& Brenner, T. (2004). Empirical Calibration of Simulation Models. In Papers on Economics and Evolution, \#0410, Jena: Max Planck Institute for Research into Economic Systems.

Woodward, J. (1989). Data and phenomena. Synthese, 79, 393-472. 